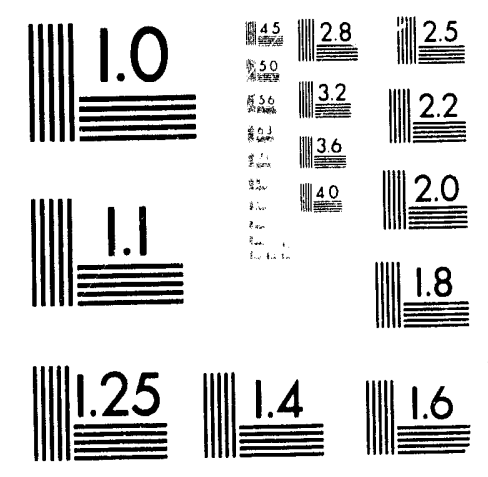




\title{
Workforce Transition Modeling Environment User's Guide
}

\author{
E. J. Stahlman \\ M. A. Oens \\ R. E. Lewis
}

October 1993

Prepared for the U.S. Department of Energy under Contract DE-ACO6-76RLO 1830

Pacific Northwest Laboratory

Operated for the U.S. Department of Energy

by Battelle Memorial Institute 


\section{Summary}

The Pacific Northwest Laboratory (PNL) was tasked by the U.S. Department of Energy Albuquerque Field Office (DOE-AL) to develop a workforce assessment and transition planning tool to support integrated decision making at a single DOE installation. The planning tool permits coordinated, integrated workforce planning to manage growth, decline, or transition within a DOE installation. The tool enhances the links and provides commonality between strategic, programmatic, and operations planners and human resources. Successful development and subsequent complex-wide implementation of the model also will facilitate planning at the national level by enforcing a consistent format on data that are now collected by installations in corporate-specific formats that are not amenable to national-level analyses.

The workforce assessment and transition planning tool, the Workforce Transition Modeling Environment (WFTME), co.ssists of two components: the Workforce Transition Model and the Workforce Budget Constraint Model. The Workforce Transition Model, the preponderant of the two, assists decision makers identify and evaluate alternatives for transitioning the current workforce to meet the skills required to support projected workforce requirements. The Workforce Budget Constraint Model helps estimate the number of personnel that will be affected by a given workforce budget increase or decrease and assists in identifying how the corresponding hirings or layoffs should be distributed across the Common Occupation Classification System (COCS) occupations.

This user's guide describes the use and operation of the WFTME. This includes the functions of modifying data and running models, interpreting output reports, and an approach for using the WFTME to evaluate various workforce transition scenarios. 


\section{Contents}

Summary $\ldots \ldots \ldots \ldots \ldots \ldots \ldots \ldots \ldots \ldots \ldots \ldots \ldots \ldots \ldots$ iii

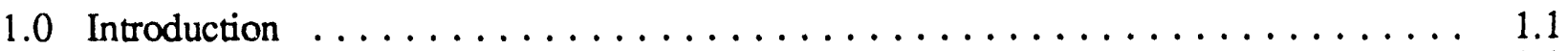

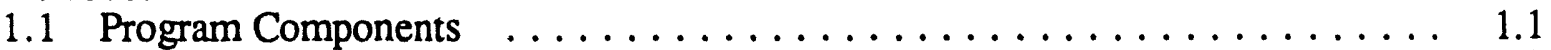

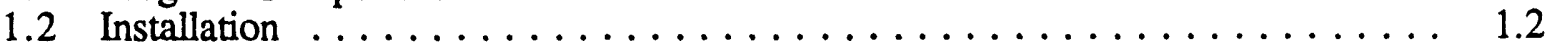

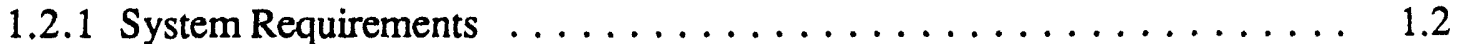

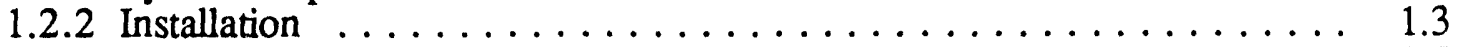

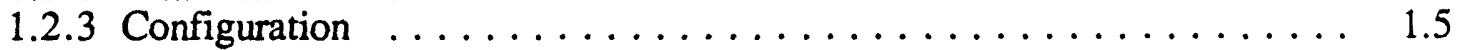

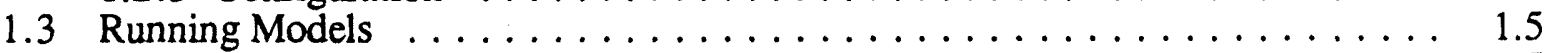

1.4 Related Reading . . . . . . . . . . . . . . . . . . . . 1.5

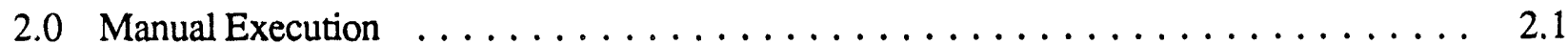

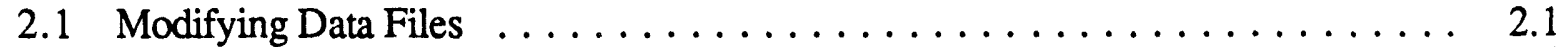

2.2 Running Models Manually $\ldots \ldots \ldots \ldots \ldots \ldots \ldots \ldots \ldots \ldots \ldots$

2.3 Loading Manually Updated .WK1 Files into the WFTME $\ldots \ldots \ldots \ldots \ldots . . . .4$

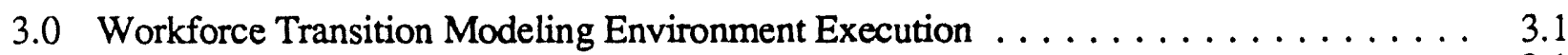

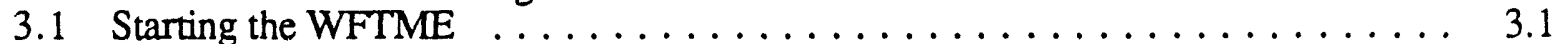

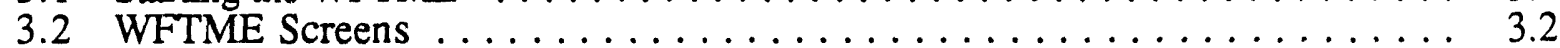

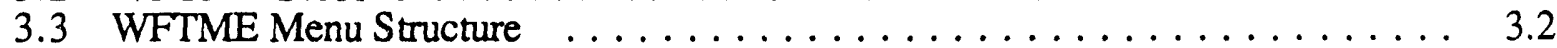

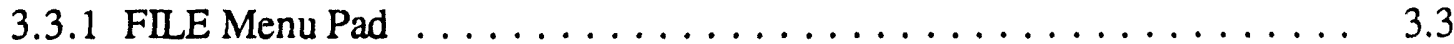

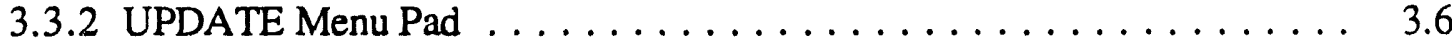

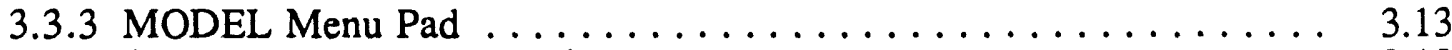

3.4 Modifying Data and Running Models $\ldots \ldots \ldots \ldots \ldots \ldots \ldots \ldots \ldots$

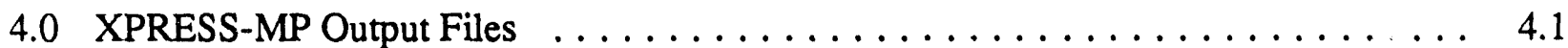

4.1 Output Field Descriptions $\ldots \ldots \ldots \ldots \ldots \ldots \ldots \ldots \ldots \ldots \ldots \ldots \ldots \ldots \ldots$

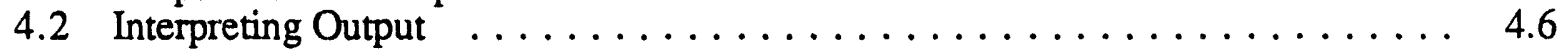

5.0 Developing Scenarios and Performing Analysis $\ldots \ldots \ldots \ldots \ldots \ldots \ldots$

5.1 Workforce Transition Mode! Formulation $\ldots \ldots \ldots \ldots \ldots \ldots \ldots \ldots \ldots$

5.2 Workforce Transition Model Scenario Development $\ldots \ldots \ldots \ldots \ldots \ldots \ldots . \ldots . \ldots . \ldots$

5.2 .1 Initial Run - Unconstrained Baseline . . . . . . . . . . . . . . 5.2

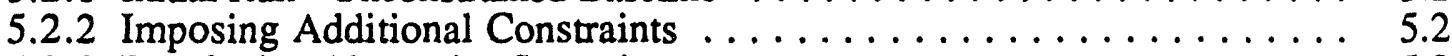

5.2 .3 Developing Alternative Scenarios $\ldots \ldots \ldots \ldots \ldots \ldots \ldots \ldots \ldots \ldots$

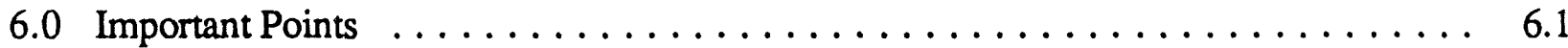

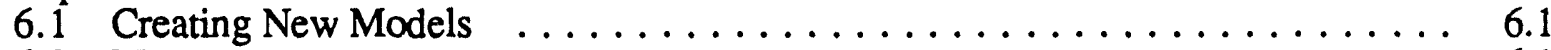

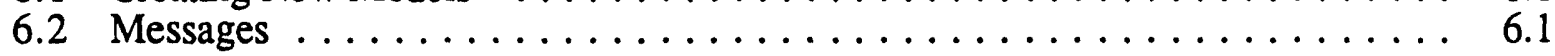

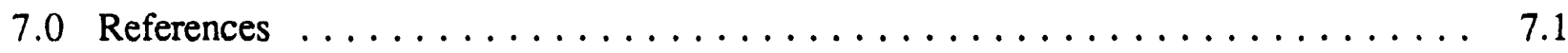




\section{Figures}

1.1 Workforce Transition Modeling Components $\ldots \ldots \ldots \ldots \ldots \ldots \ldots$

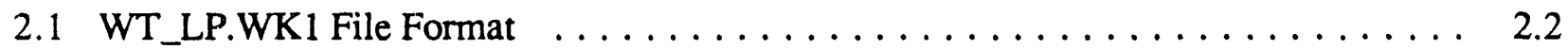

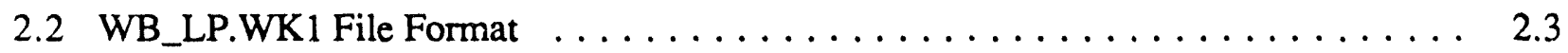

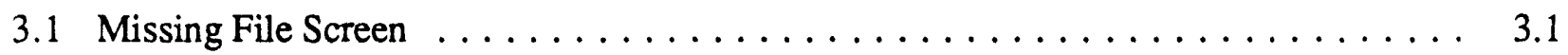

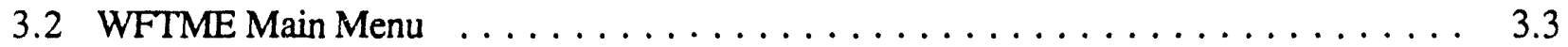

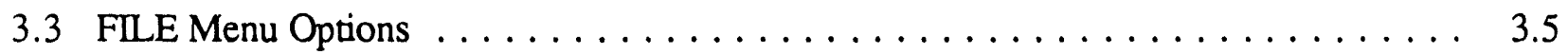

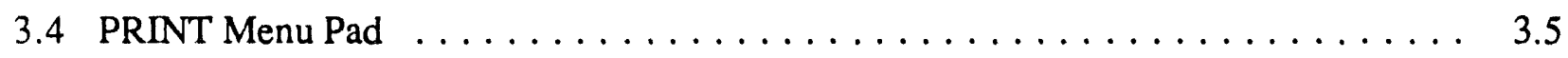

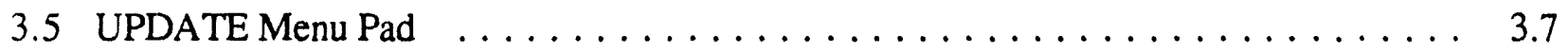

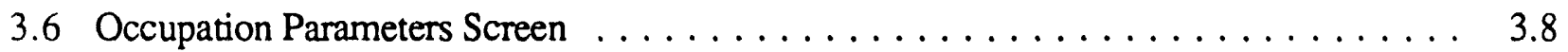

3.7 Modify Training Parameters Screen $\ldots \ldots \ldots \ldots \ldots \ldots \ldots$

3.8 Budget Pararneters Screen $\ldots \ldots \ldots \ldots \ldots \ldots \ldots \ldots \ldots \ldots \ldots \ldots \ldots$

3.9 EM FTE Distribution Parameters Screen $\ldots \ldots \ldots \ldots \ldots \ldots . \ldots \ldots$

3.10 EM Source Term Parameters Screen $\ldots \ldots \ldots \ldots \ldots \ldots \ldots . \ldots \ldots$

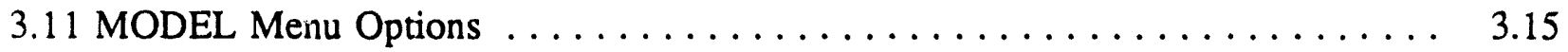

3.12 IMPORT/EXPORT Menu Options $\ldots \ldots \ldots \ldots \ldots \ldots \ldots$

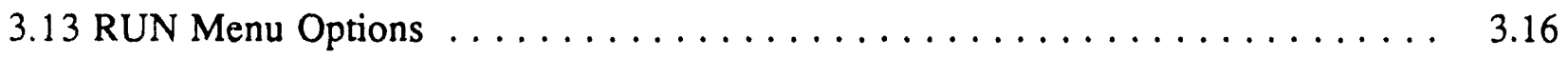

3.14 VIEW DATA Menu Options $\ldots \ldots \ldots \ldots \ldots \ldots \ldots \ldots \ldots \ldots \ldots \ldots$ 


\section{Tables}

1.1 Workforce Transition Modeling Environment Installation Files: C:IWFTM $\ldots \ldots$

1.2 Workforce Transition Modeling Environment Installation Files: C:IWFTMMMODEL1 1.4

1.3 Workforce Transition Modeling Environment Installation Files: C:IWFTMNPRESS 1.5

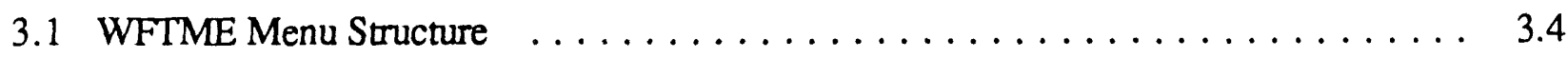

3.2 Occupation Parameters Screen Minimum and Maximum Values $\ldots \ldots \ldots$

3.3 Training Parameters Screen Minimum and Maximum Values . . . . . . . . 3.10

3.4 Budget Parameters Screen Minimum and Maximum Values $\ldots \ldots \ldots \ldots . \ldots$

3.5 EM FTE Distribution Parameters Screen Minimum and Maximum Values $\ldots \ldots .12$

3.6 EM Source Terms Parameters Screen Minimum and Maximum Values $\ldots \ldots .14$

4.1 Comprehensive Report Column Names $\ldots \ldots \ldots \ldots \ldots \ldots \ldots$

4.2 Summary Report Column Names $\ldots \ldots \ldots \ldots \ldots \ldots \ldots \ldots \ldots$

4.3 Example Output Interpretation Parameters $\ldots \ldots \ldots \ldots \ldots$

4.4 Initial LP Output File $\ldots \ldots \ldots \ldots \ldots \ldots \ldots \ldots \ldots \ldots \ldots \ldots \ldots \ldots \ldots \ldots$

4.5 LP Output File $($ with $X 0102$ Cost $=24000) \quad \ldots \ldots \ldots \ldots \ldots$

4.6 LP Output File $($ with $\mathrm{H} 02$ Cost $=35000) \ldots \ldots \ldots \ldots$ 


\subsection{Introduction}

The Workforce Transition Modeling Environment (WFTME) is a workforce assessment and transition planning tool. The WFTME consists of two models. The primary model is the Workforce Transition Planning Model for which the tool is named. The second model is the Workforce Budget Constraint Model. These models are described in the Workforce Transition Model for DOE-AL Non-Nuclear Reconfiguration, PNL-8920 (Stahlman and Lewis 1993). The purpose of this document is to explain how to use the WFTME.

\subsection{Program Components}

Figure 1.1 displays the four components required to run the workforce linear programming (LP) models. The LP package used to run the models is XPRESS-MP. Input to the package is composed of two files: the model file and the input data file. The model files are installationindependent and thus allow all installations to use the same model. The data files, which provide the coefficients to the models, are installation-dependent and will reflect the unique demographic characteristics of each installation.

The model file is an XPRESS-MP macro written in the XPRESS-MP modeling language and contains the variables, constraints, and objectives function of the model. The model files for both the Workforce Transition Model and the Workforce Budget Constraint Model are provided as part of this package and should not be modified.

Caution: The model files are XPRESS-MP marros. A thorough knowledge of XPRESS-MP and its masro language are nece'ssary to modify the code. Corrupted model files will either cause the model to fail or result in incorrect answers. If the files are accidently modified, replace them with the files from the distribution disk.

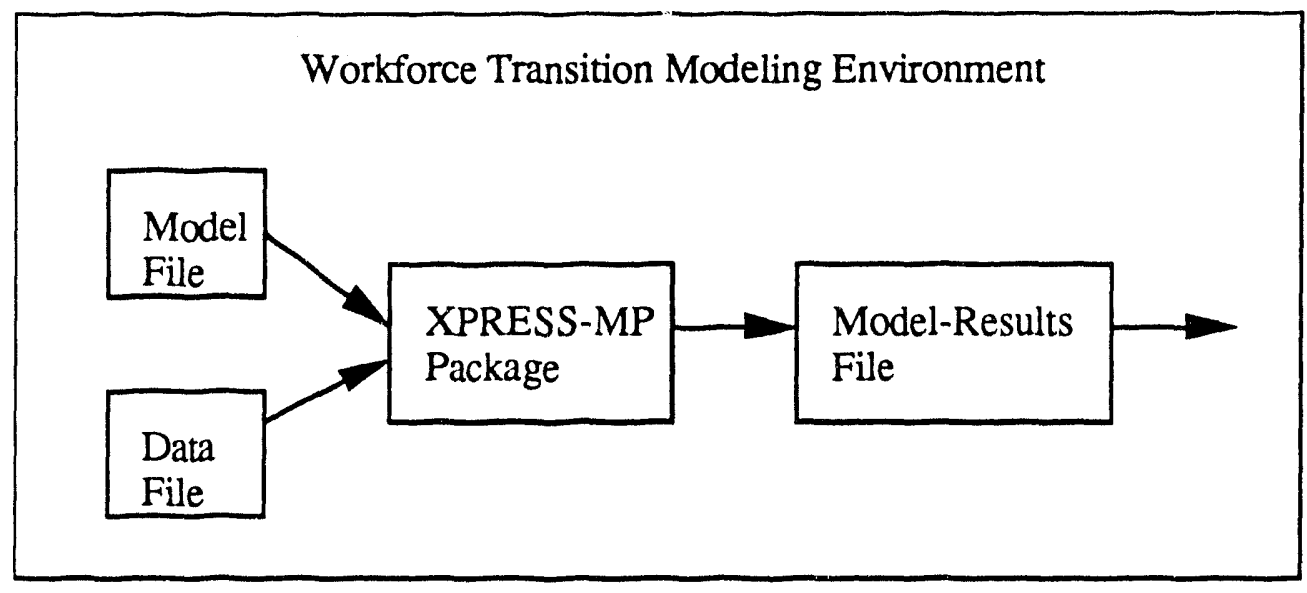

Figure 1.1. Workforce Transition Modeling Components 
The data file contains the values of the coefficients of each variable in the model file. These values are generally installation-specific; that is, they need to be specified by each installation. Generic versions of the data files are provided for both models. The user need only modify the appropriate values in either file using either a spreadsheet package or the accompanying WFTME program. Upon execution, XPRESS-MP constructs the LP model from the model file and the data file. The model is then optimized, and the optimal solution is placed into the results file, the fourth component.

The WFTME is a Windows application that integrates and simplifies these components through a menu-driven environment. Through the WFTME, the user may view or modify data, execute the model, and view the model results. These functions also may be performed directly by using a spreadsheet package to view or modify the data, and the provided DOS batch files to run XPRESS-MP. The following documentation describes both approaches and how they may be used jointly.

\subsection{Installation}

This section describes the requirements for installing and running the WFTME and explains how to install and run it.

\subsubsection{System Requirements}

The workforce models may be run manually or through the WFTME. WFTME requirements are the following (also see system requirements for Windows):

- 80386SX processor (or higher)

- 80387SX math coprocessor

- DOS 3.1 (or later)

- $8 \mathrm{MB}$ RAM (or less if using virtual memory)

- $8 \mathrm{MB}$ hard disk space

- Microsoft Windows 3.0 or higher running in 386 enhanced mode

- Mouse.

Manual execution requirements are the following (also see system requirements for XPRESS-MP):

- 80386SX processor (or higher)

- 80387SX Math Coprocessor

- DOS 3.1 (or later)

- 4 MB RAM

- $4 \mathrm{MB}$ hard disk space

- A spreadsheet or database package capable of reading and writing.WK1 files.

XPRESS-MP also must be installed in the directory C:UXPRESSMP. See the XPRESS-MP User Guide for information about installing XPRESS-MP.

Lotus 123 or a similar package that can import and export.WK1 files will be useful if the user desires to modify the .WK1 input and output files directly without using the WFTME, although this is not recommended. 


\subsubsection{Installation}

To install the WFTME:

1. Install XPRESS-MP in the directory C:UXPRESSMP (see the XPRESS-MP User Guide)

2a. From Windows:

- Insert WFTME FOR WINDOWS Disk 1

- Select 'Run...' from the 'File' menu pad

- Type: B:ISETUP

- Insert next disk when requested

2b. From DOS:

- Insert WFTM FOR DOS Disk 2

- Type: B:ISETUP

At this point, the WFTME must be run from the File Manager. To create a WFTME icon, drag WFTM.EXE to a window outside of the File Manager and release the file. For more information about placing WFTM.EXE in a Windows Group, see the Program Manager chapter in the Windows User's Guide.

Three directories are created during installation: C:IWFTM holds the files necessary to run WFTME; C:IWFTMMODEL holds all of the data files for a model; and C:IWFTMNXPRESS holds all of the files necessary to run a model. All of these files are necessary; do not delete or modify any of them. If one is deleted or modified, reinstall it.

Table 1.1 shows the files that should be in C:IWFTM after installation. WFTM.EXE is the WFTME program; it must be run from within Windows. The remaining files are support files for WFTM.EXE.

Table 1.2 shows the files that should be in C:IWFTMMMODEL1. WT_IN.WK1 and WB_IN.WK1 are the source data files. All of the WFTME tables (.DBF files) are installed empty and are filled using the IMPORT function in the WFTME. Note that the .DBF files can only be viewed within the WFTME. Three other necessary files are not included on the installation d'sks: WT_LP.WK1, WB_LP.WK1, and WB_LIM.ASC. These are the input files for the LP model and are created by the WFTME.

All of these files are needed to run the WFTME. The subset of files needed to run the model manually are WT_LP.WK1, WB_LP.WK1, and WB_LIM.ASC. See Chapter 2.0, Manual Execution, to create these files manually.

Four other important files are WT_RESA.ASC, WT_RESP.ASC, WB_RESA.ASC, and WB_RESP.ASC. They are generated by XPRESS-MP and contain the model results.

Table 1.3 shows the files that should be in C:IWFTMNPRESS. These files are used to run the LP model. The two .BAT files may be used to run models. 
Table 1.1. WFTME Files: C:IWFTM

File

MODEL1
XPRESS
FOXRUN.PIF
FOXUSER.DBF
FOXUSER.FPT
FOXW2500.ESL
WB_JOB.FRT
WB_JOB.FRX
WE_DST.FRT
WE_DST FRX
WE_RQT.FRT
WE_RQT FRX
WFTM.EXE
WT_JOB.FRT
WT_JOB.FRX
WT_TRAIN.FRT
WT_TRAIN.FRX
W_RESA.FRT
W_RESA.FRX
W_RESP.FRT
W_RESP.FRX

Descriotion
Subdirectroy holding model files
Subdirectory holding files necessary to run models
Required PIF file
Required to run program
Required to run program
Required to run program
Required to print budget data
Required to print budget data
Required to print EM Distribution data
Required to print EM Distribution data
Required to print EM Requirements data
Required to print EM Requirements data
WFTME program
Required to print Transition data
Required to print Transition data
Required to print Transition data
Required to print Transition data
Required to print LP Results
Required to print LP Results
Required to print LP Results
Required to print LP Results

Table 1.2. WFTME Installation Files: C:IWFTMMMODEL1

File

WB_IN.WK1

WB_JOB.DBF

WB_LIM.ASC

WB_LP.WK1

WB_RESA.DBF

WB_RESP.DBF

WE_DST.DBF

WE_RQT.DBF

WT_IN.DBF

WT_IN.WK1

WT_JOB.DBF

WT_LP.WK1

WT_LP.DBF

WT_RESA.DBF

WT_RESP.DBF

WT_TRAIN.DBF

W_COCS.DBF
Description

Budget input file, only necessary if importing

Budget occupation database file, required for budget model

Budget cut value: not part of installation, created by WFTME

Input file for budget LP model: not part of installation, created by WFTME

Budget results database file, required to view data

Budget results database file, required to view data

EM FTE Distribution database file

EM FTE Requirements database file

Transition input file, only necessary if importing

Transition input file, only necessary if importing

Transition occupation database file, required for Transition model

Input file for transtion LP model: not part of installation, created by WFTME

Transition output database file, required to export

Transition results database file, required to view data

Transition results database file, required to view data

Transition training database file, required for Transition model

List of COCS occupations, necessary for both models 
Table 1.3. WFTME Installation Files: C:IWFTMXPRESS

File

WB_MODEL.BAT

WB_MODEL.MOD

WB MODEL.XIP

WT_MODEL.BAT

WT MODEL.MOD

WT_MODEL.XIP
Description

Executes Budget LP model

Budget model XPRESS-MP model file

Budget model XPRESS-MP macro file

Executes Transition LP model

Transition model XPRESS-MP model file

Transition model XPRESS-MP macro file

\subsubsection{Configuration}

No modifications are necessary to the AUTOEXEC.BAT or CONFIG.SYS other than those specified by Windows and XPRESS-MP.

\subsection{Running Models}

The models may be run either manually or through the WFTME. This section describes both methods and how they may be used jointly. Both approaches use the same basic approach:

1. Modify the input data file.

2. Run the model.

3. Browse the model output.

The WFTME provides the easiest method for entering data and running the model. The manual approach is presented, however, because the models run several orders of magnitude faster in DOS than in Windows (DOS without Windows running, not a DOS window within Windows). When execution time is critical, the best approach may be to modify the data using the WFTME, run the model in DOS, and then run the WFTME to view the results.

\subsection{Related Reading}

The WFTME employs a variety of tools and techniques beyond workforce planning.

Knowledge of these areas is useful to properly run, understand, and use these models. Useful, related reading material includes:

- XPRESS-MP User Guide, Dash Associates, Northants, United Kingdom, 1993.

(XPRESS-MP is distributed in the United States through Resource Optimization, Inc.

Knoxville, Tennessee.)

- Microsoft Windows User's Guide.

- Any Linear Programming Textbook.

NOTE: Although the models have been configured to allow the user to run them without an understanding of the principles of linear programming (LP), users should be familiar with LP to fully understand the implications of the model and the resulting output. Knowledge of LP is essential to interpreting results and performing sensitivity analysis. 


\subsection{Manual Execution}

If the model will be run exclusively through Windows, this section may be skipped.

Of the installed WFTME files, five are needed to run the model manually: WT_LP.WK1, WB_LP.WK1, WB_LIM.ASC, WB_MOD.BAT, and WT_MOD.BAT. WT_LP.WK 1 is the data file for the Workforce Transition Model, and WB_LP.WK1 and WB_LIM.ASC are the data files for the Workforce Budget Constraint Model.

\subsection{Modifying Data Files}

The data files, WT_LP.WK1 and WB_LP.WK1, may be modified in any spreadsheet or database program that can read and write. WK1 files. The format for these files was given in Figures 2.1 and 2.2. WB_LIM contains the budget reduction level associated with the budget model: it contains a single integer value between 0 and 99999999 (without commas).

NOTE: WT_LP.WK1 and WB_LP.WK1 are not installed during the installation process. They must be generated either using the WFTME Generate commands or by copying WT_IN.WK1 and WB_IN.WK1 into WT_LP.WK1 and WB_LP.WK1, respectively. A new row must then be added to each file with the column heading names according to Figures 2.1 and 2.2.

The data in WT_LP.WK1 are divided into five sections in Figure 2.1. The first two columns are the common occupation classification system (COCS) (Holmes et al. 1992) occupation code and the COCS occupation title and the next eight columns contain information specific to each occupation: Hiring Cost, Layoff Cost, Current (number in occupation), Projected (number in occupation), Min Hire (minimum number to hire in occupation), Max Hire (maximum number to hire in occupation), Min Lay (minimum number to lay off in occupation), and Max Lay (maximum number to lay off in occupation).

The next 240 columns contain parameters controlling training between pairs of occupations. The column labels are generated automatically by the WFTME when the output file is generated and are limited to 10 characters. The column headings are based on the COCS occupation codes instead of the occupation names.

Columns Description

$1-2$ - COCS code and COCS occupation title.

$3-10$ - Occupation specific information.

11-91 - The costs to train from an occupation in column 2 to each other occupation. This value must be a positive value and should be an integer between 0 and 99999 .

$92-172$ - The minimum number of people that must be trained from an occupation in column 2 to each other occupation. These columns allow minimum training rec . irements to be enforced. The initial and default value for these columns is 0 . This number should be between 0 and 9999 and must be less than the corresponding maximum number of people that may be trained. 


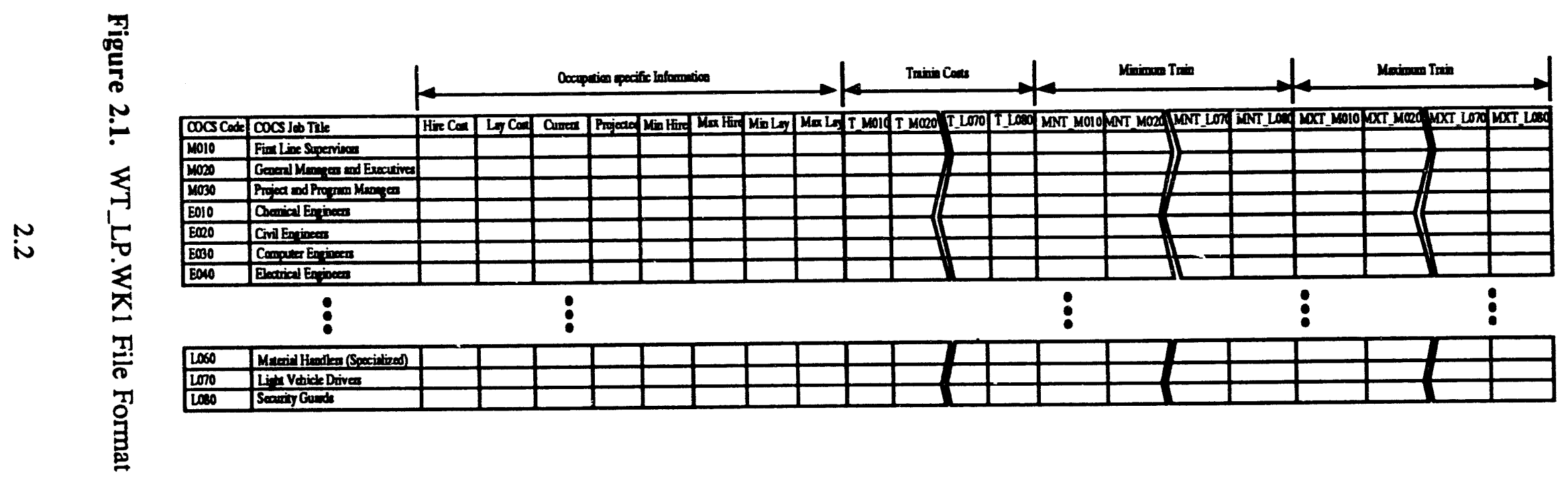




\begin{tabular}{|l|l|l|l|l|l|l|}
\hline COCS Code & COCS Occupation Title & Salary & Other Cost & Total Cost & Minimum RIF & Maximum RIF \\
\hline M010 & First Line Supervisors & & & & & \\
\hline M020 & General Managers and Executives & & & & & \\
\hline M030 & Project and Program Managers & & & & & \\
\hline E010 & Chemical Engineers & & & & & \\
\hline E020 & Civil Engineers & & & & & \\
\hline E030 & Computer Engineers & & & & & \\
\hline E040 & Electrical Engineers & & & & & \\
\hline
\end{tabular}

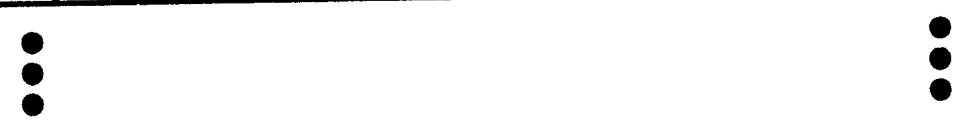

\begin{tabular}{|l|l|l|l|l|l|l|}
\hline L060 & Material Handlers (Specialized) & & & & & \\
\hline L070 & Light Vehicle Drivers & & & & & \\
\hline L080 & Security Guards & & & & & \\
\hline
\end{tabular}

Figure 2.2. WB_LP.WK1 File Format

173 - 253 - The maximum number of people that may be trained from an occupation in column 2 to each other occupation. These columns provide an upper limit to the number of people that may train from one occupation to another. The initial and default value for these columns is 9999 . This number should be between 0 and 9999 and must be greater than the corresponding minimum number of people that must be trained.

Most of the numeric fields have certain limitations including minimum and maximum values. The WFTME ensures these limitations are not violated. Check Tables 3.2 through 3.6 for limiting values.

NOTE: Certain training costs are set to 888888 to indicate forbidden transitions that will not occur because of high cost or exceptionally long completion time. When the WFTME encounters a training cost of 888888 , the user is not allowed to modify associated training values. Furthermore, the XPRESS-MP linear program is configured to not evaluate these transitions regardless of the training cost. Therefore, changing values associated with forbidden transitions has no effect on the model.

Caution: The LP input data files are large and cumbersome; WT_LP is 80 rows by 246 columns. Careful tracking of rows and columns is required to ensure the appropriate cells are modified. It is difficult to find an incorrect value once it is entered. The WFTME avoids this problem through strict data access control.

Caution: Updates to WT_LP.WK1 and WB_LP.WK1 do not update the corresponding values in the WFTME database. Once the output files are modified outside of the WFTME, two versions of the data exist that cannot be directly resolved.

Caution: Failure to observe these formats will cause the model to either fail or generate incorrect answers. 


\subsection{Running Models Manually}

The transition model may be run using WT_MODEL.BAT, and the budget constraint model may be run using WB_MODEL.BAT. These batch files require that the .WK1 data files be located in C:IWFTMXPRESS.

To run the transition model:

Type> C:IWFTMXPRESSIWT_MODEL "path"

where "path" is the location of the WT_LP.WK1 file including the trailing "Y". For example,

C:IWFTMNPRESSTWT_MODEL C:IWFTMMMODEL1

To run the budget model from C:IWFTMMPRESS:

Type> C:IWFTMXPRESSTWB_MODEL "path"

where "path" is the location of the WB_LP.WK1 file including the trailing " $\backslash$ ". For example,

C:IWFTMXPRESSIWB_MODEL C:IWFTMMODELII

Output files generated by XRESS-MP will be placed in the directory specified by "path".

If the .BAT files are used to run the model, the results files will be named WT_RESA.ASC and WT_RESP.ASC for the transition model, and WB_RESA.ASC and WB_RESP.ASC for the budget constraint model. The two files, *_RESA.ASC and *_RESP.ASC, represent two forms of the results. *_RESA.ASC is a comprehensive report, and *- RESP.ASC is a summary report. Both formats are described in Section 4.0 and in the XPRESS-MP User Guide (1993). (1993).

For additional information on running the model manually, see the XPRESS-MP User Guide

\subsection{Loading Manually Updated .WK1 Files into the WFTME}

Manually modified output files are not forever excluded from the WFTME. They may be loaded into the WFTME with slight modification. The only difference between the WFTME input and output .WK1 files is the column heading row in W?_LP.WK1.

NOTE: The .WK1 file format is not a completely standard format; there are several versions. The two files may be saved in different format versions so they may differ slightly in file size and format. For example, if WT_IN.WK1 is created and saved using Lotus 123 v2.0 for DOS and WT_LP.WK1 is edited using Lotus 123 v1.1 for Windows, they will be saved in slightly different formats. The data are not affected.

To use a modified output data file in the WFTME:

1. Create a new model directory.

2. Copy all of the necessary model files into the new directory. 
3. Open the modified W?_LP.WK1 in a spreadsheet where ? is ' $T$ ' or 'B,' depending on the model.

4. Delete the first row containing the column headings.

5. Save the file in.WK1 format using the name W?_IN.WK1.

7. Start WFTME.

8. Import the W?_IN.WK1 file using the Import Transition or Import Budget command. 


\subsection{Workforce Transition Modeling Environment Execution}

This section discusses modifying data and running models in the WFTME.

\subsection{Starting the WFTME}

The WFTME is a Windows application. It behaves similarly to Windows, so most Windows actions should work in the WFTME. To start the WFTME:

1. Open the file manager and double-click on the WFTM.EXE file icon, or

2. Double-click on the WFTM icon if it resides in a Windows group.

Once the Welcome Screen appears, hit any key to continue. Upon start-up, the WFTME asks the user to select a directory containing a model. Select the directory containing the model to be used. If any of the required files are not found in the selected directory, a warning screen appears that lists the missing files (see Figure 3.1). A checked box means the necessary files are missing or empty; these may be either.DBF or.WK1 files. In most cases, importing, generating output files, or running the model will remedy the problem. If the .DBF files are missing, they must be replaced.

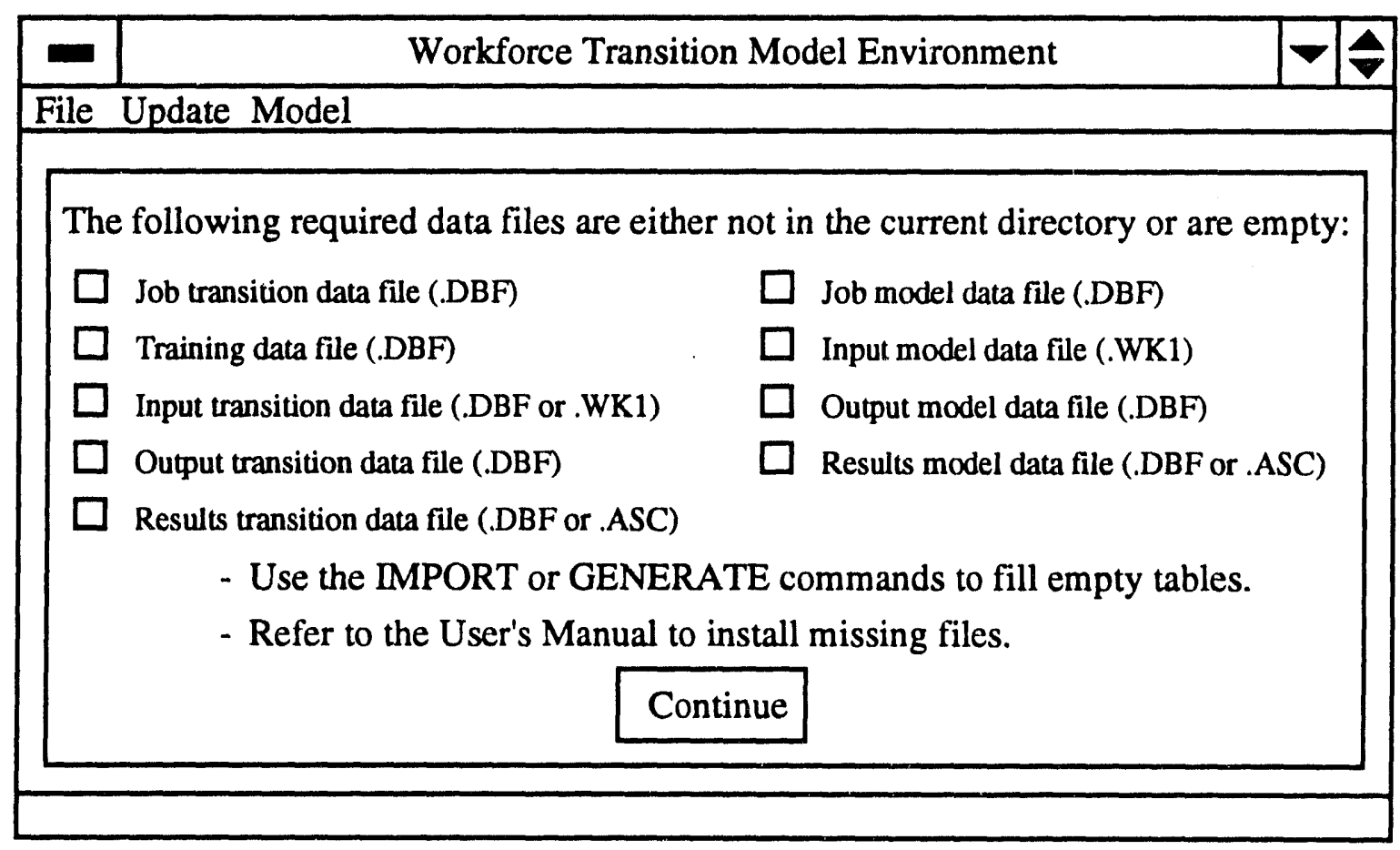

Figure 3.1. Missing File Screen 
The following list describes the meaning of the data files checklist:

- If all of the boxes are checked, the current directory does not contain a model. Use the Change Current Directory command to move to a directory with a model.

- If either of the input boxes is checked, a model may not be imported; the W?_IN.WK1 file is missing. The import function is only used when a WK1 model is created or modified outside of the WFTME and is to be loaded into the WFTME. If the loaded model is the working model, the import function is not needed.

- If either of the output boxes is checked, an XPRESS-MP data file does not exist and the model cannot be run. Generating the output file should remedy this problem.

- If one or both of the results boxes is checked, one or both of the models has not been run. Running the model will make these files available.

- If any other boxes are checked or the above remedies do not work, necessary files are missing and must be replaced from the installation disk.

NOTE: The check boxes may be checked or unchecked by clicking on them with the mouse. This has no effect on the program or the availability of the files.

NOTE: To see if a missing file is created by either running the model or generating an output file, use the Change Current Directory command and reselect the current directory. This will redisplay the missing file screen.

If any of these problems cannot be resolved, a .DBF file is probably missing. Use the File Manager or DOS to look at the directory containing the model. If one or more of the .DBF files listed in Table 1.2 is missing, replace it from the Master Disk. This should solve the problem.

\subsection{WFTME Screens}

Figure 3.2 displays the WFTME main screen. The two important areas are the Menu Bar and the Status Bar. The Menu Bar is used to perform specific functions. If files required for a particular function are not available, the corresponding menu item is disabled.

NOTE: When certain menu items are selected, a new screen will appear and the Menu Bar will disappear. The Menu Bar will reappear after the screen is closed.

Across the bottom of the screen, the Status Bar displays messages regarding the current selection. Messages include menu item descriptions, data entry ranges, and delay notices.

\subsection{WFTME Menu Structure}

The WFTME main menu has three options: FILE, UPDATE, and MODEL (see Figure 3.2). Table 3.1 displays the entire menu structure. The following sections describe each menu function. 


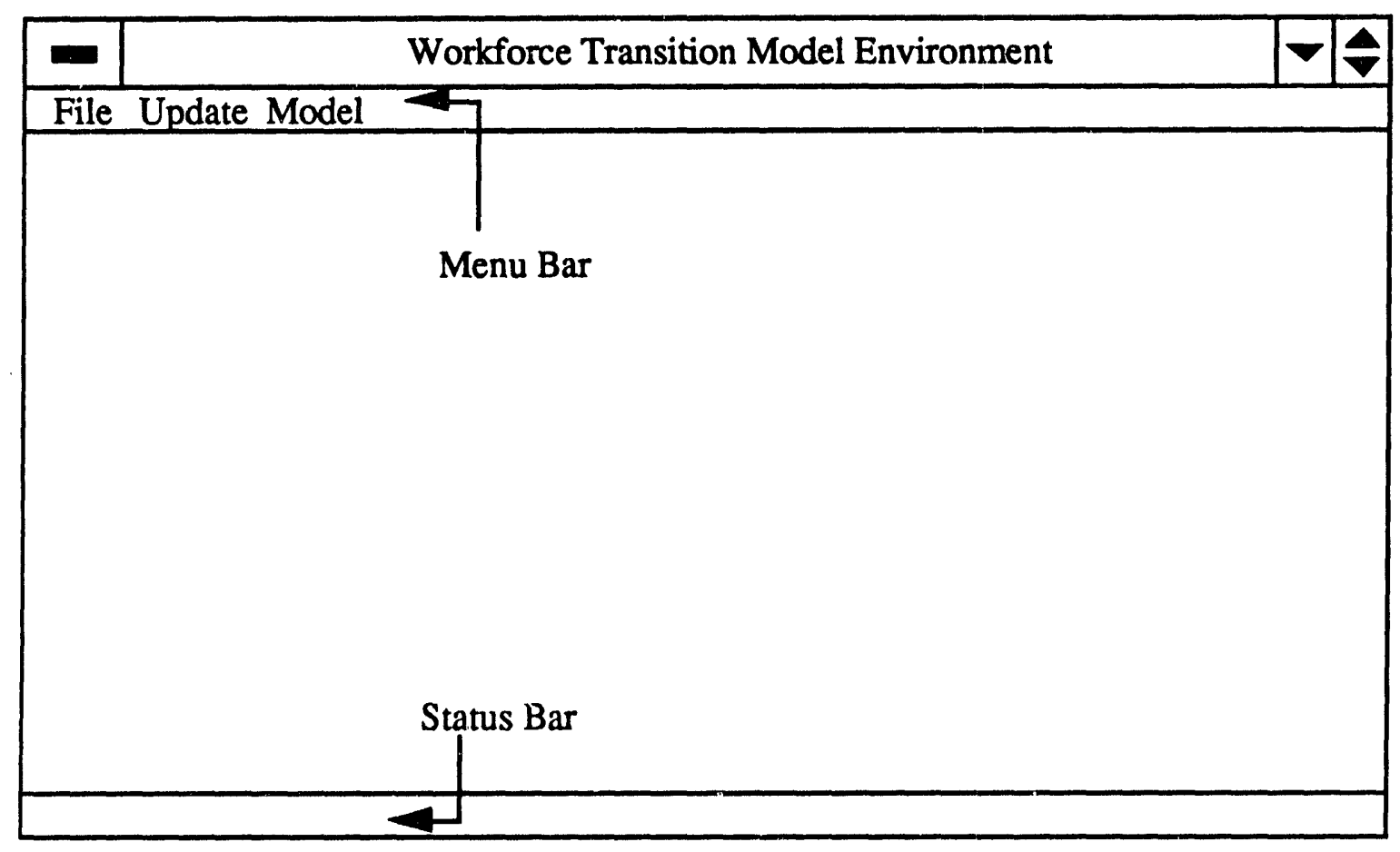

Figure 3.2. WFTME Main Menu

\subsubsection{FILE Menu Pad} options.

FILE allows the user to perform general file operations. Figure 3.3 displays the FILE menu

\section{Change Working Directory Menu Option}

CHANGE WORKING DIRECTORY changes the current directory to the selected directory and attempts to load a model. If one or more required files are missing, a window will appear specifying what files are missing (see Figure 3.1). If all of the boxes are checked, the selected directory does not contain a model.

\section{Print Menu Option}

PRINT is used to print one of WFTME's five reports (see Figure 3.4):

TRANSITION OCCUPATION DATA

OCCUPATION TRAINING DATE

SUMMARY TRANSITIONS RESULTS

COMPLETE TRANSITIONS RESULTS

BUDGET MODEL DATA
SUMMARY BUDGET RESULTS

COMPLETE BUDGET RESULTS

EM DISTRIBUTION

EM REQUIREMENTS 
Table 3.1. WFTME Menu Structure

FILE

CHANGE WORKING DIRECTORY

PRINT

TRANSITION OCCUPATION DATA

OCCUPATION TRAINING DATE

SUMMARY TRANSITIONS RESULTS

COMPLETE TRANSITIONS RESULTS

BUDGET MODEL DATA

SUMMARY BUDGET RESULTS

COMPLETE BUDGET RESULTS

EM DISTRIBUTION

QUIT

EM REQUIREMENTS

UPDATE

OCCUPATION PARAMETERS

TRAINING PARAMETERS

BUDGET PARAMETERS

EM FTE DISTRIBUTION

EM SOURCE TERM REQUIREMENTS

CALCULATE FTES

MODEL

IMPORT/EXPORT

IMPORT TRANSITION

IMPORT BUDGET

GENERATE TRANSITION

GENERATE BUDGET

IMPORT TRANSITION RESULTS

RUN

IMPORT BUDGET RESULTS

TRANSITION MODEL

BUDGET MODEL

VIEW DATA

Selecting a report immediately starts printing all pages; there is no option of specifying which pages to print. Select the CANCEL button to stop printing.

NOTE: WT_LP.WK1 and WB_LP.WK1, the two input files to XPRESS-MP, cannot be printed. Use a spreadsheet package to print them.

NOTE: The TRAINING DATA REPORT and COMPLETE TRANSITION RESULTS REPORT are about 124 pages each. Printing may be easier if the .ASC or.WK1 file is imported into a spreadsheet program. 


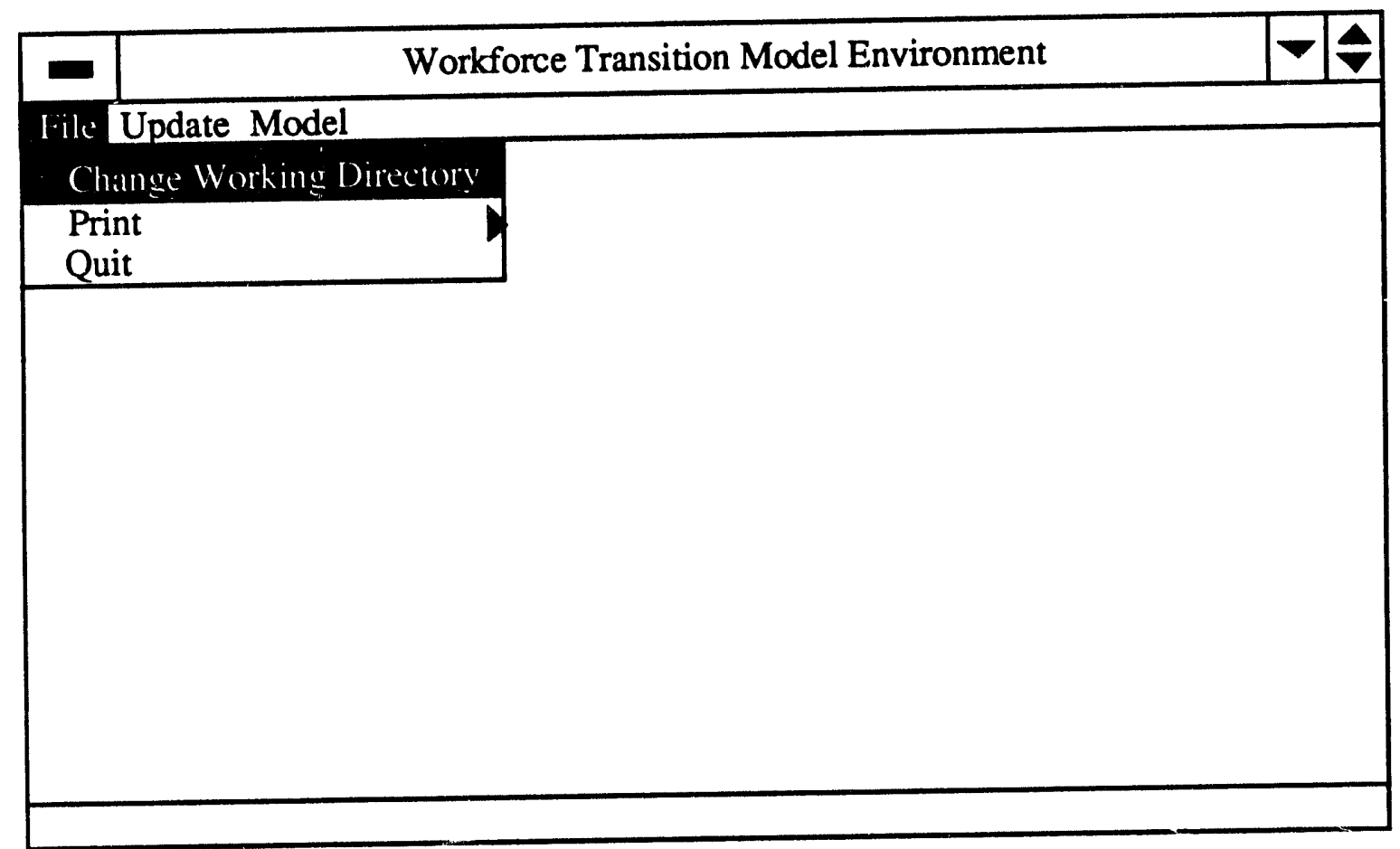

Figure 3.3. FILE Menu Options

\begin{tabular}{|c|c|c|}
\hline $\mathbf{m}$ & \multicolumn{2}{|l|}{ Workforce Transition Model Environment } \\
\hline \multicolumn{3}{|c|}{ Iilc Update Model } \\
\hline \multicolumn{3}{|c|}{ Change Working Directory } \\
\hline Print & Occupation Transition Data & \\
\hline Quit & ()ccupation Thaiming Datri & \\
\hline & $\begin{array}{l}\text { Summary Transition Results } \\
\text { Complete Transition Results } \\
\text { Budget Model Data } \\
\text { Summary Budget Results } \\
\text { Complete Budget Results } \\
\text { EM Distribution } \\
\text { EM Requirements }\end{array}$ & \\
\hline
\end{tabular}

Figure 3.4. PRINT Menu Pad 


\section{Quit Menu Option}

QUIT terminates the WFTME and returns control to Windows.

\subsubsection{UPDATE Menu Pad}

There are two ways to view and modify data: through individual data screens or through a spreadsheet-like interface. The menu options under the UPDATE menu pad allow the user to access data from each table one row at a time; the spreadsheet approach, the View menu option under the Model menu pad, presents the entire table at once. Both approaches enforce similar data entry constraints. The advantage of the spreadsheet interface is the ability to quickly traverse the table and to see its contents all at once. The advantage of the individual data screens is that the displayed data are limited to a single occupation. The two methods are equivalent.

Figure 3.5 displays the six menu selections under the UPDATE menu pad. The transition model has three types of data, occupation specific data, training data between two occupations, and Environmental Restoration and Waste Management full-time equivalents (EM FTE) data. The budget model only has occupation specific data. When one of the update options is selected, the corresponding update screen appears.

Within each screen, use either the mouse, arrow keys, or tab key to select values to modify (tab and arrow keys work best). To modify a set of values:

1. Select an occupation from the Occupation Title pop-up menu.

2. Select a field and enter its new value. Repeat for each field to be modified.

3. Select the Save button to save the changes.

Caution: Be sure to save changes after modifying each occupation's values. If a new occupation title is selected before the changes are saved, the changes are lost.

4a. To modify the next occupation, go to step 1 .

4b. Select the DONE button to stop modifying data and exit the Set Parameters screen. If changes are not saved when the DONE button is selected, a dialog box will appear asking if the changes should be discarded. Select the CONTINUE button to discard the changes or the RETURN button to return to the screen where the changes can be saved.

\section{Occupation Parameters Menu Option}

Figure 3.6 displays the Occupation Parameters screen that appears when Occupation Parameters is selected from the UPDATE menu. This screen is used to view or modify the following data:

Occupation Title: The occupation for which data is displayed.

Hiring Costs: Total costs associated with hiring an employee in the occupation. 


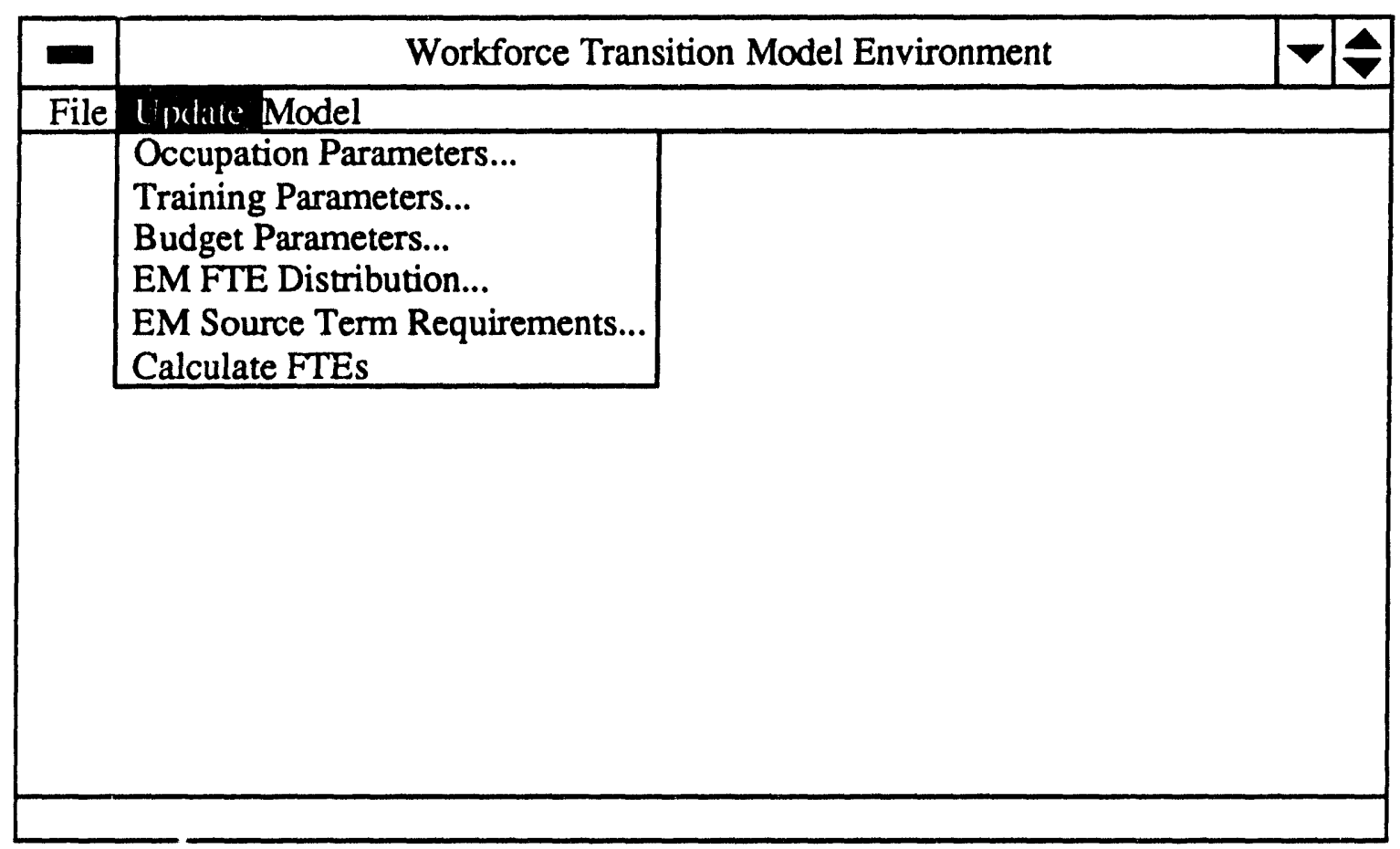

Figure 3.5. UPDATE Menu Pad

Layoff Costs: $\quad$ Total costs associated with laying off an employee in the occupation.

Current:

Projected: The current number of employees in the occupation.

Minimum hire: The minimum number of employees of this type that may be hired.

Maximum hire: The maximum number of employees of this type that may be hired.

Minimum layoff: The minimum number of employees of this type that may be laid off.

Maximum layoff: The maximum number of employees of this type that may be laid off.

Select the occupation to modify using the pop-up menu labeled "Occupation title". The minimum and maximum values for each field are given in Table 3.2. The WFTME also enforces these restrictions:

- Minimum layoff must be less than or equal to Current.

- Maximum hire must be greater than or equal to Minimum hire.

- Maximum layoff must be greater than or equal to Minimum layoff.

\section{Training Parameters Menu Option}

Figure 3.7 displays the Training Parameters screen that appears when Training Parameters is selected from the UPDATE menu. This screen is used to view or modify the following data concerning training from one occupation to another:

Train from: $\quad$ The occupation from which an employee could train.

Train to: The occupation to which the employee could train. 


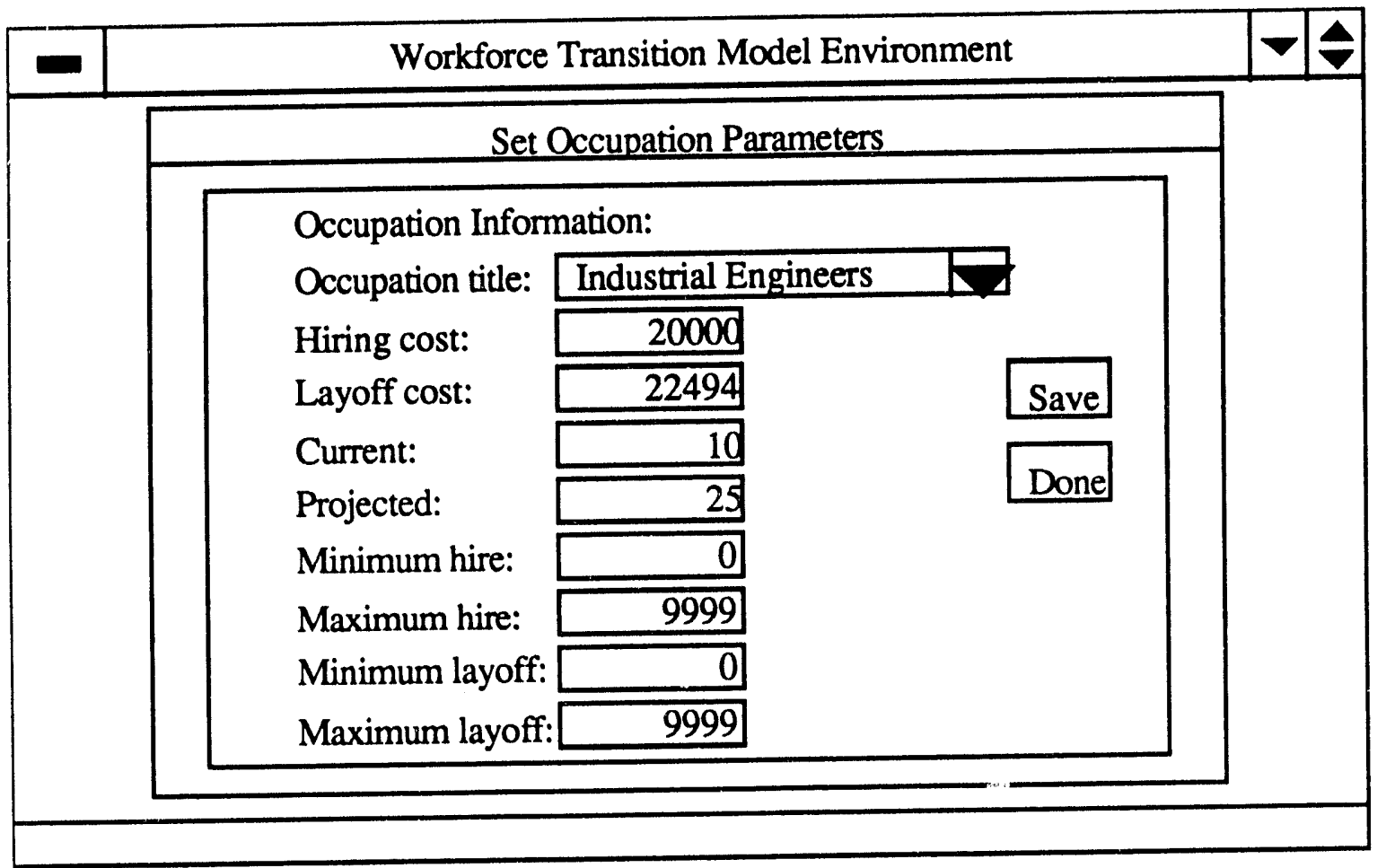

Figure 3.6. Occupation Parameters Screen

Table 3.2. Occupation Parameters Screen Minimum and Maximum Values

Attribute

Hiring cost

Layoff cost

Current

Projected

Minimum hire

Maximum hire

Minimum layoff

Maximum layoff
Minimum

0

0

0

0

0

0

0

0
Maximum

99999

99999

9999

9999

9999

9999

9999

9999 


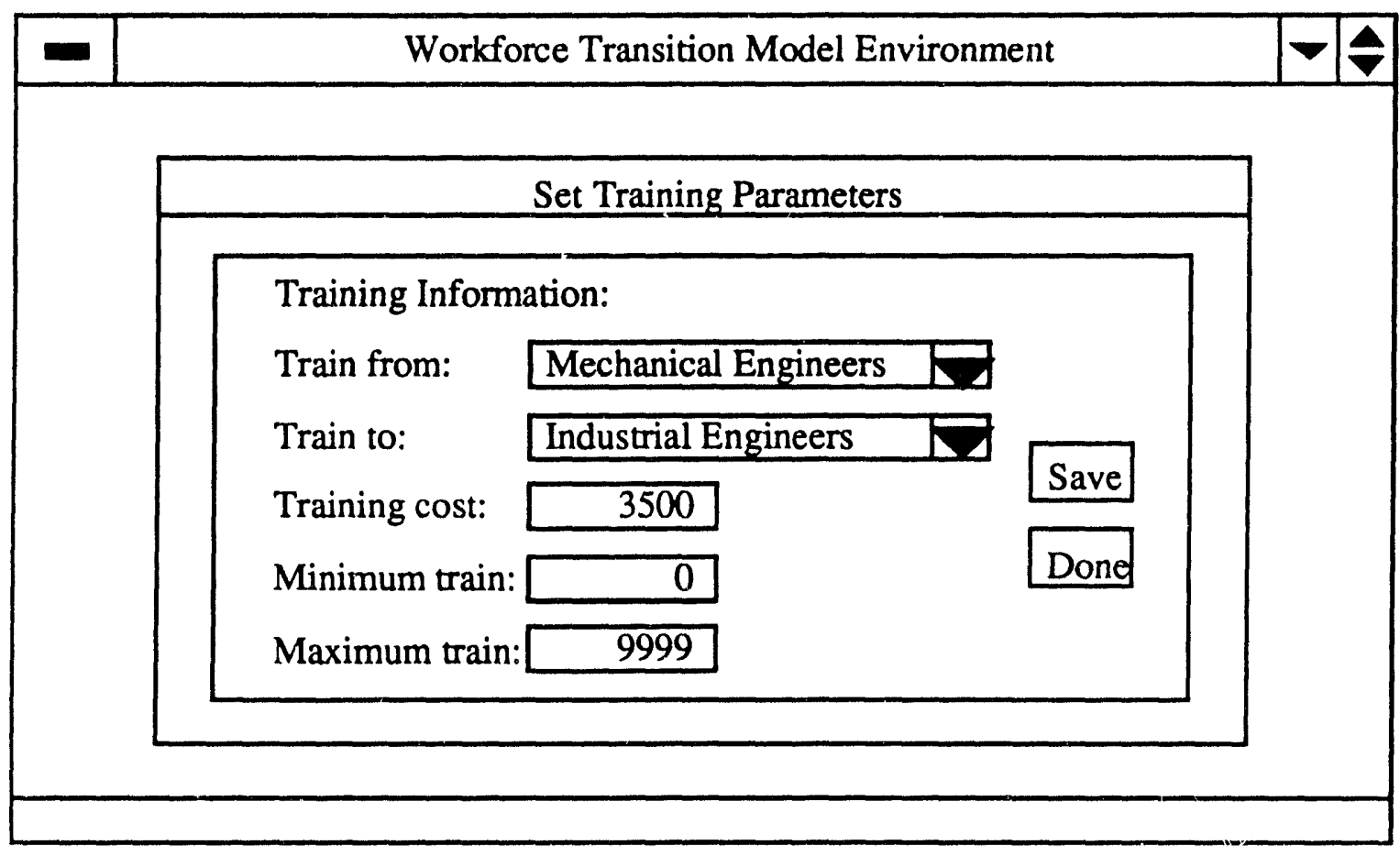

Figure 3.7. Modify Training Parameters Screen

Training cost: The cost to train an employee from the first occupation to the second occupation.

Minimum train: The minimum number of employees that will train from the first occupation to the second occupation.

Maximum train: The maximum number of employees that are allowed to train from the first occupation to the second occupation.

The "Train from" and "Train to" occupations are selected using the two pop-up menus. The Minimum train and Maximum train parameters allow the user to evaluate prescribed scenarios by explicitly controlling the transitions among occupations. The minimum and maximum values for each field are given in Table 3.3. The WFTME also enforces these restrictions:

- Maximum train must be greater than or equal to Minimum train.

NOTE: The value for training from occupation $A$ to occupation $B$ is not necessarily the same as the value for training from occupation $B$ to occupation $A$.

Certain transitions are forbidden because they are inordinately expensive or will take longer than the scope of the planning period to complete. These transitions are identified with a training cost of 888888. Because this value is larger than the maximum training cost listed in Table 3.3, the WFTME will not allow the user to modify this value. Thus, the user may not make forbidden transitions allowable. Furthermore, the XPRESS-MP linear programming models are configured to ignore these transitions regardless of the training cost. 
Table 3.3 Training Parameters Screen Minimum and Maximum Values

\begin{tabular}{ccc} 
Attribute & Minimum & Maximum \\
\cline { 3 - 3 } Training cost & 0 & 99999 \\
Minimum train & 0 & 9999 \\
Maximum train & 0 & 9999
\end{tabular}

\section{Budget Parameters Menu Option}

Figure 3.8 displays the Modify Budget Parameters screen that appears when Modify Budget Parameters is selected from the UPDATE menu. This screen is used to view or modify the following occupation-related data:

Occupation Title: The occupation for which data is displayed.

Salary: Average salary of employees in the occupation.

Other Costs: Aggregate costs other than salary the user may want to include related to laying off an employee.

Minimum RIF: The minimum number of employees that are laid off in the occupation.

Maximum RIF: The maximum number of employees that may be laid off. If the total number in this field for all of the occupations is too small, an infeasible solution will result because not enough employees can be laid off to meet the required budget cut.

Savings Required: The savings required from the RIF.

Caution: Savings Required is occupation-independent. That is not dependent on the occupation displayed on the screen. This value may be changed when any occupation is displayed and will not change when a new occupation is chosen.

Select the occupation to modify using the pop-up menu labeled "Occupation title". The field "Savings required" specifies the budget reduction that must be achieved. The minimum and maximum values for each field are given in Table 3.4. The WFTME also enforces these restrictions:

- Maximum RIF must be greater than or equal to Minimum RIF. 


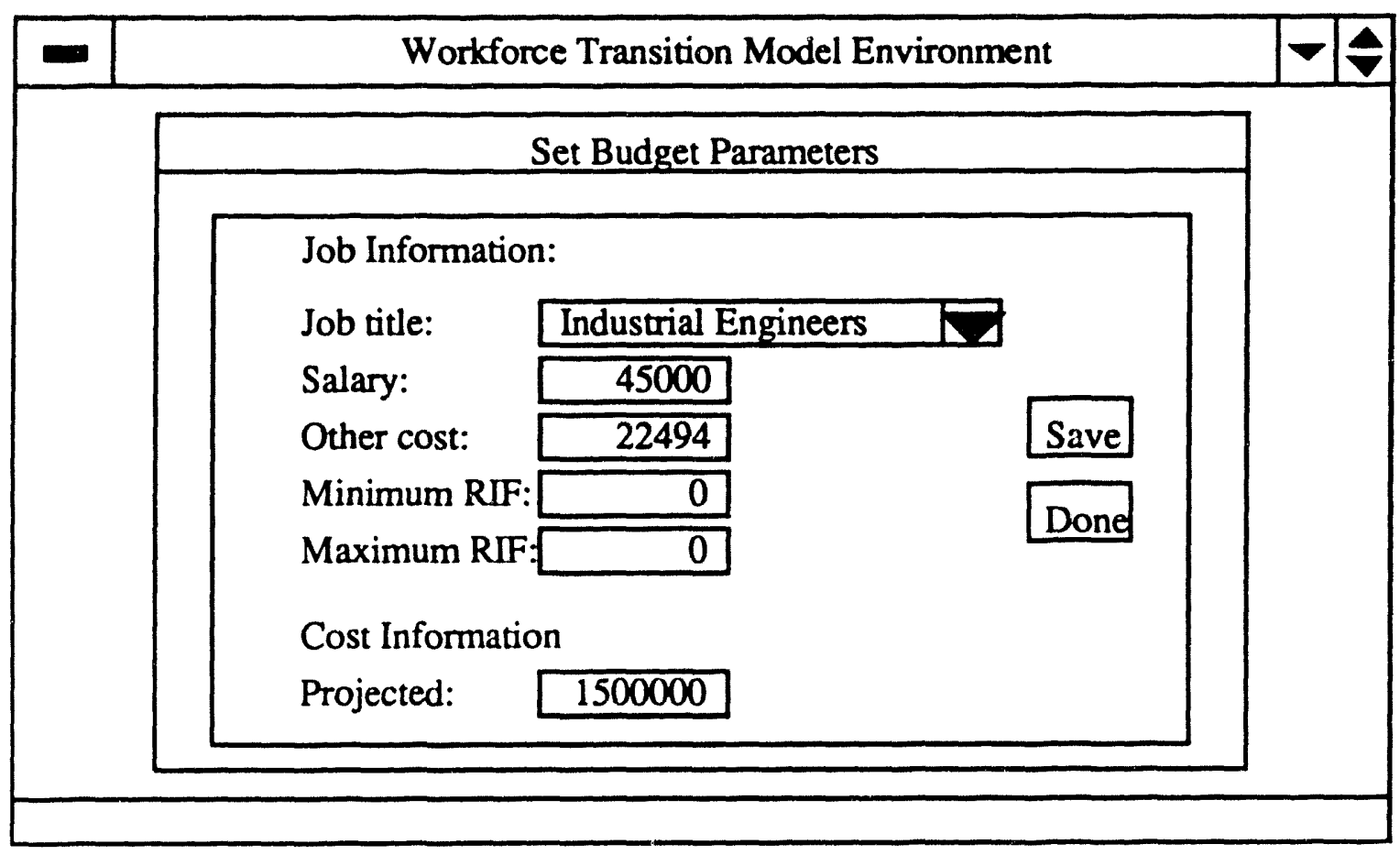

Figure 3.8. Budget Parameters Screen

Table 3.4. Budget Parameters Screen Minimum and Maximum Values

\begin{tabular}{|c|c|c|}
\hline Attribute & Minimum & Maximum \\
\hline Salary & 0 & \\
\hline Other cost & 0 & \\
\hline Minimum RIF & 0 & 1 \\
\hline Maximum RIF & 0 & 1 \\
\hline Savings required & 0 & 999999 \\
\hline
\end{tabular}

\section{EM FTE Distribution Menu Option}

Figure 3.9 displays the Set EM FTE Distribution Parameters screen that appears when EM FTE Distribution is selected from the Update menu. This screen is used to view or modify the following occupation-related data:

Occupation: The occupation for which data is displayed.

Percent of FTEs: The percentage of FTEs required for a remediation project that are of the specified occupation type. Note that the sum of percentages for all occupations must equal 100 .

Minimum FTEs: The minimum number of employees of the specified occupation type that are required for a remediation project. 
Select the occupation to modify using the pop-up menu labeled "Occupation". The minimum and maximum values for each field in Figure 3.\% are given in Table 3.5.

Caution: Values are provided in the model for percent of FTEs and Minimum FTEs. These values are based on the literature and experience of remediation experts. Do not change these values unless you have better estimates for your installation.

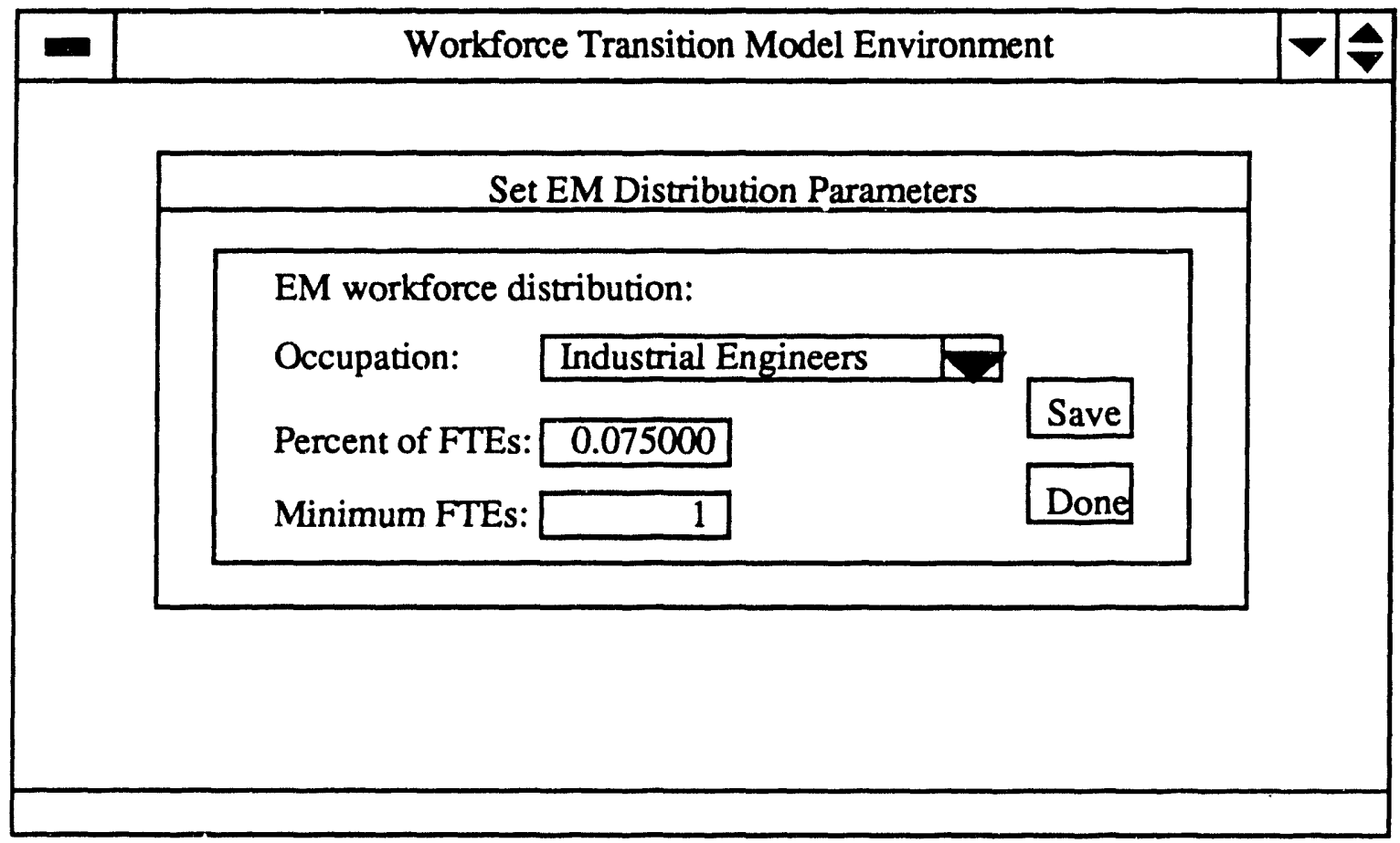

Figure 3.9. EM FTE Distribution Parameters Screen

Table 3.5. EM FTE Distribution Parameters Screen Minimum and Maximum Values

Attribute

Percent of FTEs Minimum FTEs
Minimum

0.0

0
Maximum

1.0

9999

\section{EM Source Term Requirements Menu Option}

Figure 3.10 displays the Set EM Source Term Requirements Parameters screen that appears when EM Source Term Requirements is selected from the Update menu. This screen is used to view or modify the following source-term-related data:

Source term:

General category of waste created during installation operation. 
FTEs/m3: The average number of FTEs required to remediate one cubic meter of waste. This number is estimated from previous remediation experience.

Volume $\left(\mathrm{m}^{3}\right)$ : $\quad$ The volume of waste of the source term type. This may be the total volume of waste at the installation or the volume associated with a particular project. The scope must be consistent, however, across all source terms.

Caution: Values are provided in the model for FTEs $/ \mathrm{m}^{3}$. These values are based on the literature and experience of remediation experts. Do not change these values unless you have better estimates for your installation.

Select the source term to modify using the pop-up menu labeled "Source term". The minimum and maximum values for each field in Figure 3.10 are given in Table 3.6.

\section{Calculate FTEs}

The final option on the Update menu pad is Calculate FTEs, which calculates the number of employees needed in each COCS occupation based on the EM FTE distribution and EM source term requirements. The calculated values are stored in the Projected column of the Transition Occupation table. This allows easy evaluation of alternative EM scenarios by varying the volume of each type of source term.

This option first sums the products of the FTEs $/ \mathrm{m}^{3}$ and the corresponding Volumes of each type of waste. This yields the total number of required FTEs to remediate all specified waste. The number of FTEs is then multiplied by the Percent of FTEs specified in the EM Distribution table to calculate the number of FTEs per COCS occupation. This number is rounded to the nearest integer. If the final number is less than Minimum FTEs for the corresponding occupation, the number of employees is set to Minimum FTEs. This value is then entered into the Projected column of the Transition Occupation table.

\subsubsection{Model Menu Pad}

The MODEL menu pad is used to import and export data files, run a model, and view the data files (see Figure 3.11).

\section{Import/Export Menu Option}

IMPORT/EXPORT is used to import and export data to and from .WK1 files. IMPORT allows data to be entered or modified in a spreadsheet program and then imported into the WFTME. EXPORT creates the.WK1 files used as data input files for the XPRESS-MP LP package. 


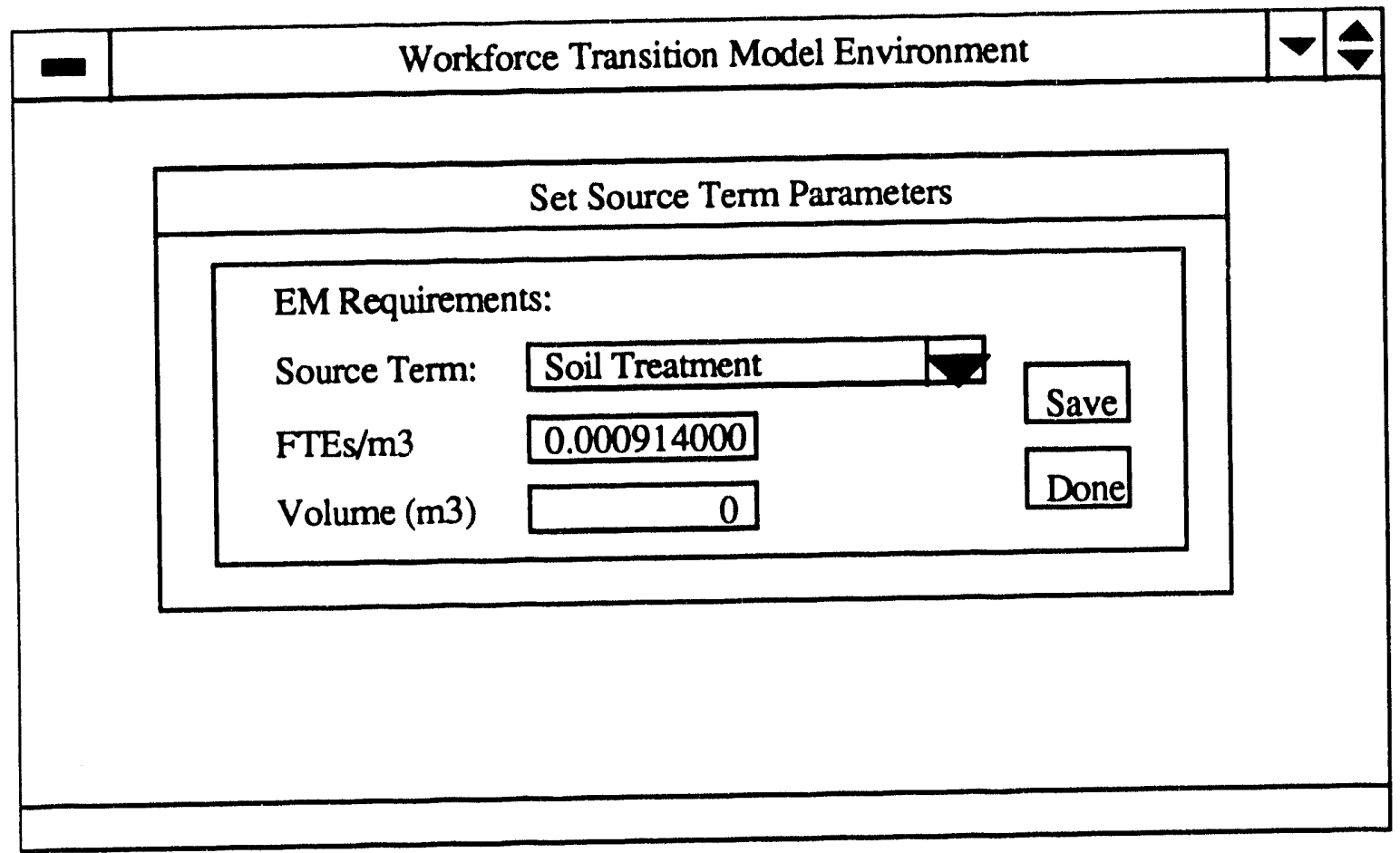

Figure 3.10. EM Source Term Parameters Screen

Table 3.6. EM Source Terms Parameters Screen Minimum and Maximum Values

\begin{tabular}{lrr}
\multicolumn{1}{c}{ Attribute } & Minimum & Maximum \\
\hline FTEs $/ \mathrm{m}^{3}$ & 0.0 & 999 \\
Volume $\left(\mathrm{m}^{3}\right)$ & 0 & 999999
\end{tabular}

Caution: The import and export file formats are not identical and are not interchangeable. The export file has one extra row for column headings.

The six IMPORT/EXPORT options are (see Figure 3.12):

- IMPORT TRANSITION: This imports the transition model.WK1 file WT_IN.WK1. Any changes made using the WFTME since the last import will be lost. To save a model, create a new model directory and copy the necessary files.

- IMPORT BUDGET: This imports the occupation model .WK1 file WB_IN.WK1. Any changes made using the WFTME since the last import will be lost. To save a model, create a new model directory and copy the necessary files.

- GENERATE TRANSITION: This creates the transition model.WK1 file WT_LP.WK1. This will delf ie the previous output data file.

- GENERATE BUDGET: This creates the budget constraint model.WK1 file WB_LP.WK1. This will delete the previous output data file. 


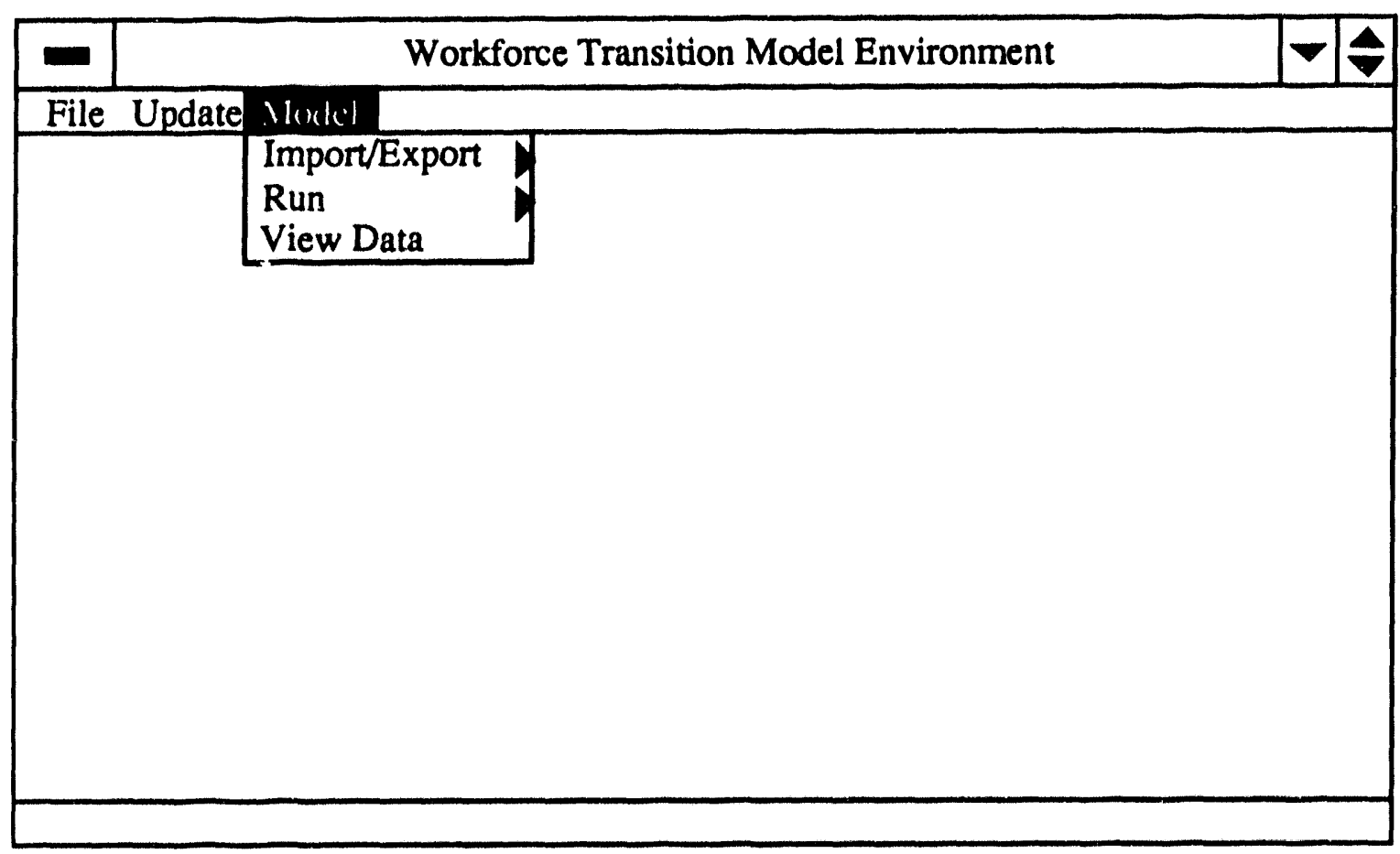

Figure 3.11. MODEL Menu Options

- IMPORT TRANSITION RESULTS: This option imports the workforce transition output files (WT_RESA.ASC and WT_RESP.ASC) created when the linear program is run. Importing of these files is only necessary when the linear program is run manually. If the models are run from within the WFTME Lsing the RUN TRANSITION MODEL menu option, the files are automatically imported.

- IMPORT BUDGET RESULTS: This option imports the budget constraint output files (WB_RESA.ASC and WB_RESP.ASC) created when the linear program is run. Importing of these files is only necessary when the linear program is run manually. If the models are run from within the WFTME using the RUN BUDGET MODEL menu option, the files are automatically imported.

\section{Run Menu Option}

RUN executes the XPRESS-MP using one of the two models (see Figure 3.13). The two run options are

- TRANSITION MODEL: This runs the transition model.

- BUDGET MODEL: This run the budget model. 


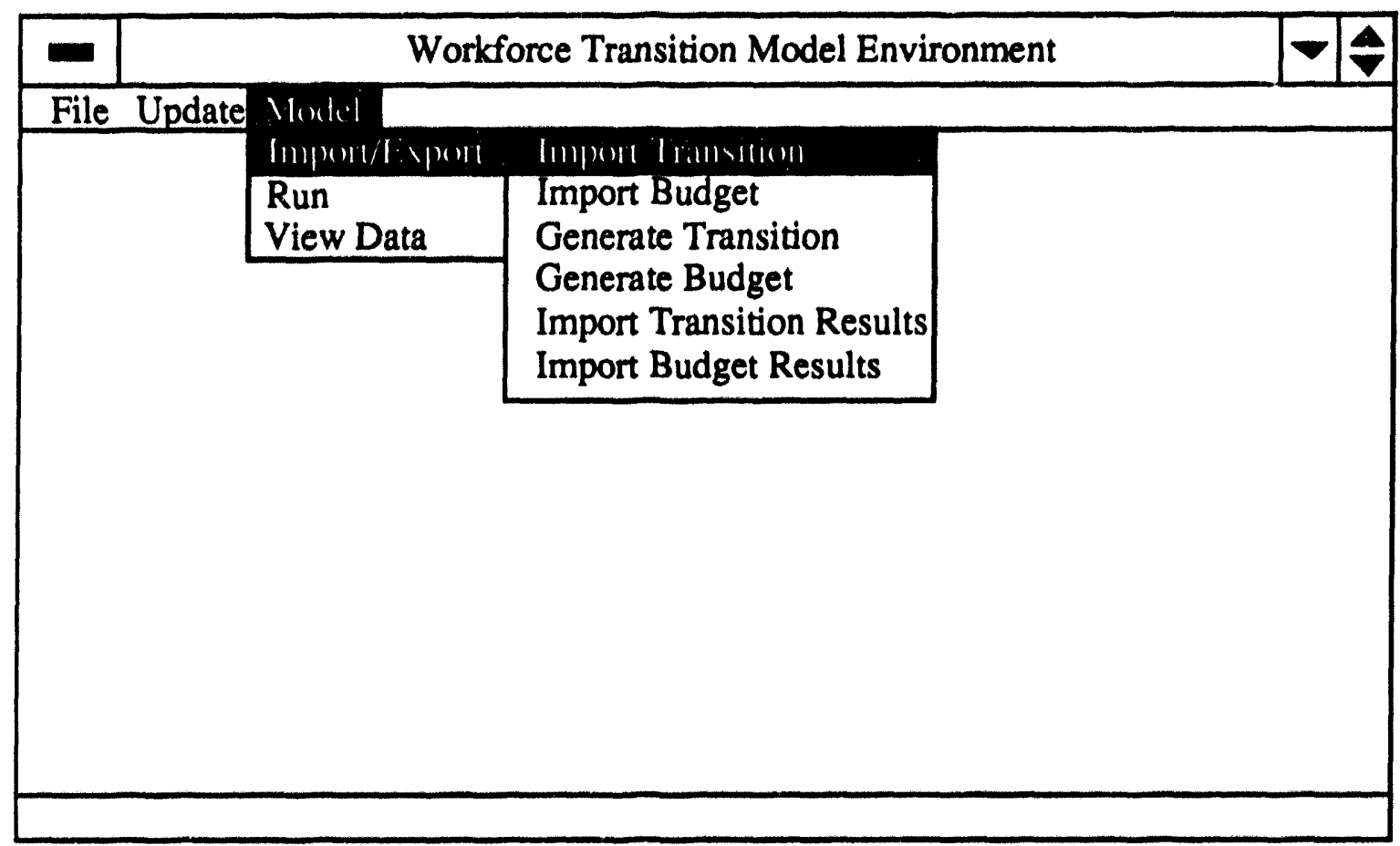

Figure 3.12. IMPORT/EXPORT Menu Options

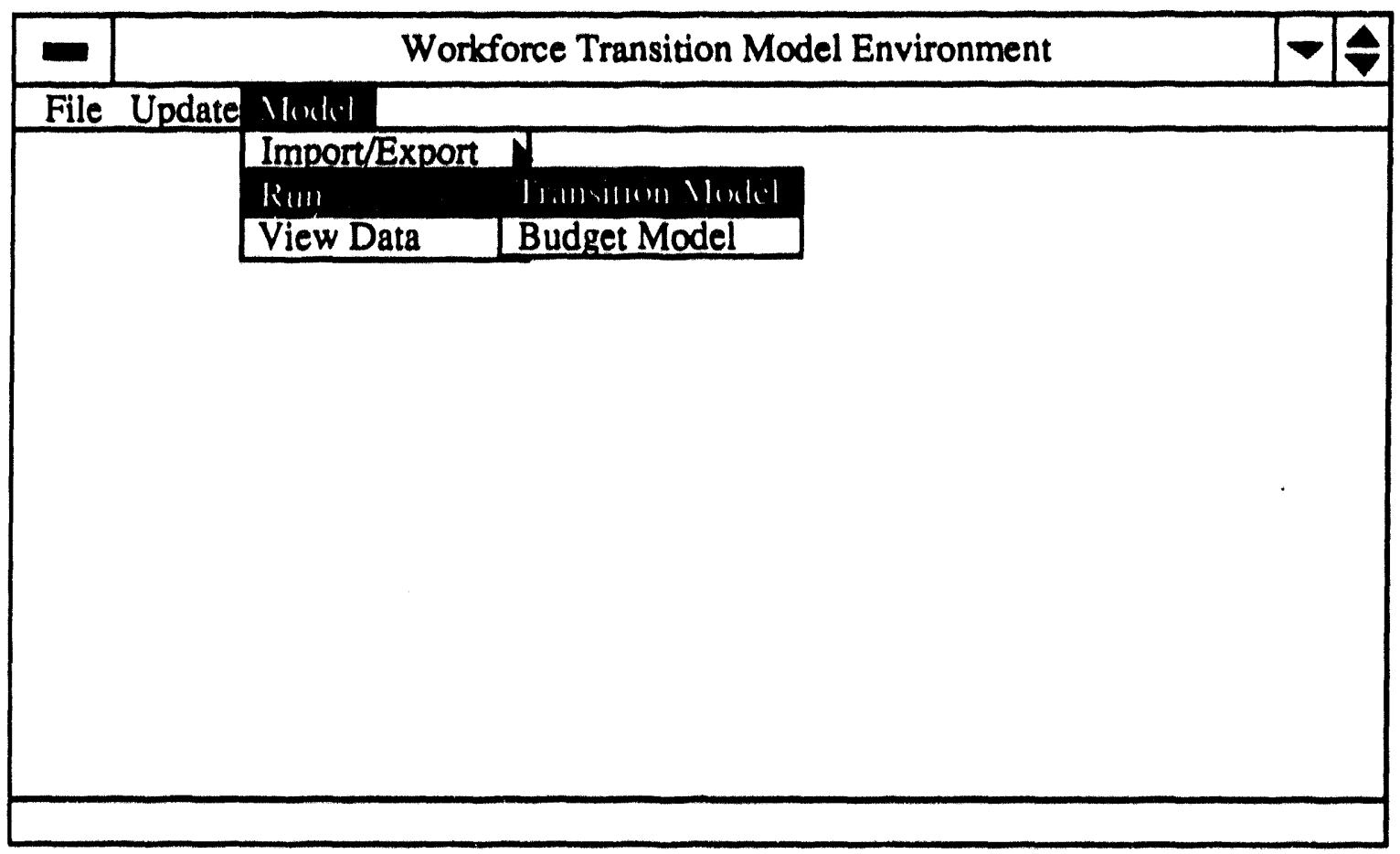

Figure 3.13. RUN Menu Options 


\section{View Data Menu Option}

Figure 3.14 displays the View Data Tables screen that appears when View Data is selected from the MODEL menu pad. Choosing one of the tables will open a browse window, allowing viewing of the data in the table. The eight tables that may be viewed are

- Transition Occupation

- Transition Training

- Transition Output

- Transition Comprehensive Result

- Transition Summary Results

- Budget Occupation

- Budget Comprehensive Results

- Budget Summary Results. window:

In addition, the values in the following tables may be modified directly in the browse

- Transition Occupation

- Transition Training

- Budget Occupation.

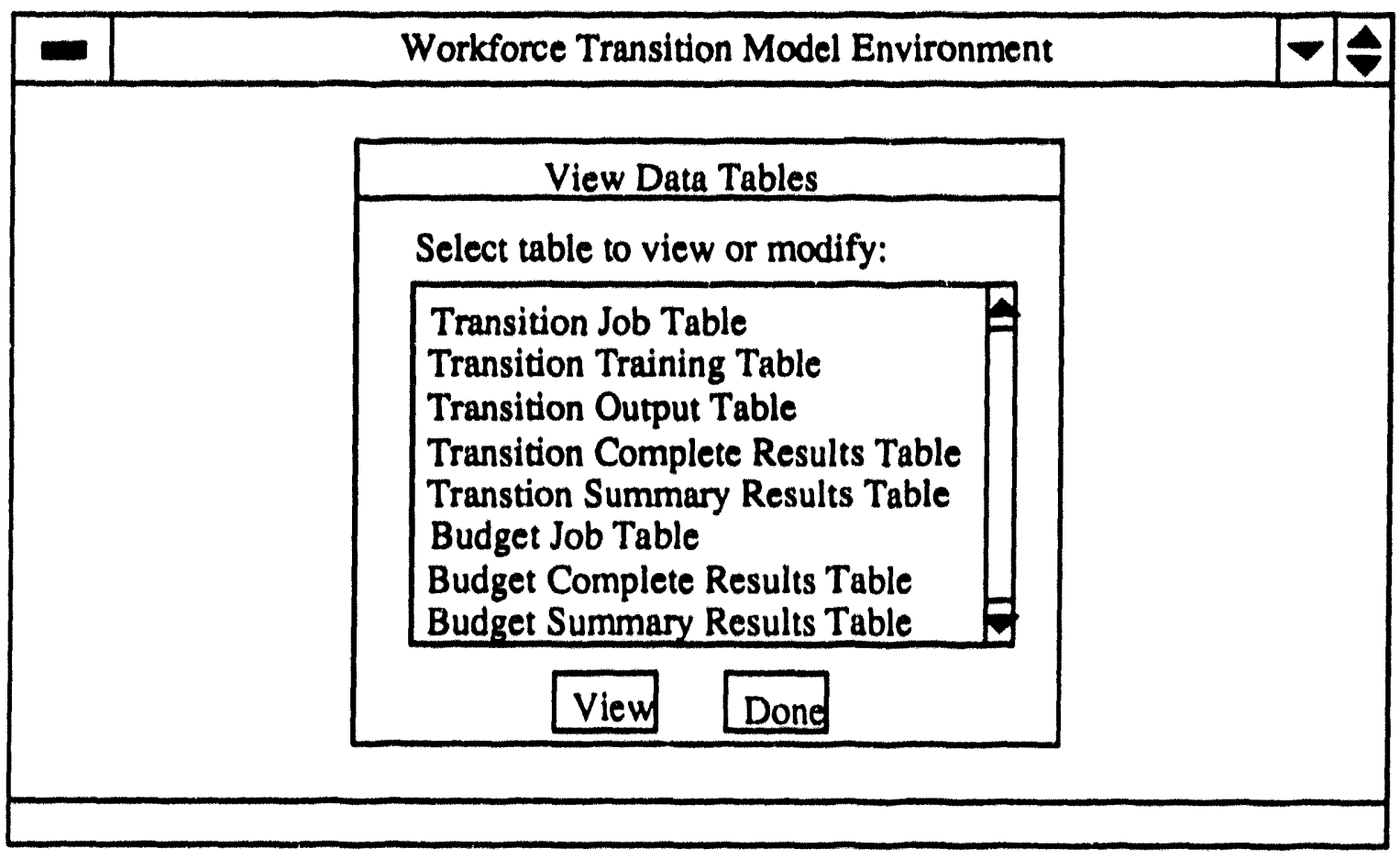

Figure 3.14. VIEW DATA Menu Options 
This is an alternative method of updating values to the Modify Parameters menu options under the UPDATE menu pad. Most of the data restrictions in the corresponding Modify Parameters screens are enforced in the browse window. The other tables may only be viewed; the data may not be modified.

The Escape key will close these windows.

\subsection{Modifying Data and Running Models}

The previous sections described the menu structure and functions of the WFTME. This section describes how to use the WFTME to modify data files, run models, and view the data.

The general procedure for using the WFTME is to:

1. Modify data.

2. Create input file for the linear program.

3. Run the linear program.

4. View results.

5. Repeat steps 1 to 4 as necessary.

When the WFTME is first run, the data files will be empty and the user will need to use the IMPORT commands to import the transition and budget data. This step only needs to be repeated if the user decides to modify the.WK1 files outside of the WFTME or the user decides to start over with a fresh database.

The menu options under the UPDATE menu pad or the VIEW option under the MODEL menu pad are used to modify data. Once the appropriate updates are complete, the EXPORT commands under the MODEL menu pad are used to generate the input files for the linear program. When the export is complete, the RUN commands under the MODEL menu pad will run the model. When the linear program is complete, the user can use the VIEW command to peruse the output. This procedure is repeated each time the user wants to run a different scenario.

Caution: Be sure to export the data before each run. If the data are not exported, the model will use an old version of the data.

Occasionally, menu options will be grayed-out. This indicates that the data required to perform those functions are not available. This is usually remedied by progressing through the steps outlined above. Initially, almost all of the functions will be grayed-out because the data files are initially empty. The IMPORT commands will instanciate all of the data files, thus allowing access to the UPDATE and EXPORT commands. Finally, once data are exported, the RUN commands become available. 


\subsection{XPRESS-MP Output Files}

Running the models, either manually or with WFTME, will generate two files:

WT_RESA.ASC and WT_RESP.ASC for the transition model and WB_RESA.ASC and WB_RESP.ASC for the budget model. If the model produces an infeasible solution only the W?_RESP.ASC files will be generated.

\subsection{Output Field Descriptions}

The WT_RESP.ASC and WB_RESP.ASC files contain a comprehensive output report and are the reports available in the WFTME. They are generated using the RTOASC command in XPRESS-MP. These reports include one row of data on every variable and constraint in the model. Thus the WT_RESP.ASC report has about 4500 rows; no small task to evaluate.

The WT_RESA.ASC and WB_RESA.ASC files are summary reports that only contain variables in the solution, generally around 40 or 50 variables. They are created using the TOASC cornmand in XPRESS-MP.

Table 4.1 lists the columns for the comprehensive-format report files. The first column lists the column name, the second column lists the abbreviated name used in the WFTME browse commands; the abbreviated names are necessary because variable names must be 10 characters or less in length. Table 4.2 lists the columns of the summary-format report files.

NOTE: Limiting Process Down Status and Limiting Process Up Status are not included in the WFTME printed output report.

Table 4.1. Comprehensive Report Column Names

\begin{tabular}{l} 
Full Name \\
\hline Sequence Number \\
Name \\
Type \\
Basis Status \\
Activity \\
Cost \\
Lower Activity \\
Unit Cost Down \\
Upper Cost \\
Limiting Process Down \\
Limiting Process Down Status \\
Upper Activity \\
Unit Cost Up \\
Lower Cost \\
Limiting Process Up \\
Limiting Process Up Status
\end{tabular}

Abbreviated Name

seq_num
name
type
basis
activity
cost
l_activity
u_cost_dwn
upper_cost
lim_dn_pro
up_status
u_activity
u_cost_up
lower_cost
lim_up_pro
low_status


Table 4.2. Summary Report Column Names

\begin{tabular}{|c|c|}
\hline Full Name & Abbreviated Name \\
\hline $\begin{array}{l}\text { Sequence Number } \\
\text { Name } \\
\text { Type } \\
\text { Basis Status } \\
\text { Activity } \\
\text { Cost } \\
\text { Lower Bound } \\
\text { Upper Bound } \\
\text { Reduced Cost } \\
\text { Righthand Side }\end{array}$ & $\begin{array}{l}\text { seq_num } \\
\text { name } \\
\text { type } \\
\text { basis } \\
\text { activity } \\
\text { cost } \\
\text { l_bound } \\
\text { u_bound } \\
\text { reduc_cost } \\
\text { rhs }\end{array}$ \\
\hline
\end{tabular}

\section{Sequence Number}

The sequence number is the row number of the variable as generated by XPRESS-MP. These numbers will generally be nonsequential because the table will be sorted by other columns. This column is not important.

\section{Name}

The variable or constraint name. The variables correspond to the data described in Chapters 2.0 and 3.0. The names are cryptic, however, because they represent the variable names generated by XPRESS-MP when it generates the model. The majority of the variables relate to the occupational names, training, hiring, laying off, and displacement. The codes are based on the COCS occupational codes. The first letter and the second two numbers are used to uniquely identify each occupation. For example,

M001 $\rightarrow \quad$ M01 or M 01, or

$\mathrm{P} 012 \rightarrow \quad \mathrm{P} 12$ or $\mathrm{P} 12$.

In the following examples, $X$ and $Y$ are the first letter of the occupational code and \#\# and ** each represent the last two digits of the occupational code.

\section{Transition Model Variables}

$\mathrm{XY}$ \#** Training variables representing training from $\mathrm{X}$ to $\mathrm{Y}^{* *}$. Thus the variable representing training from mechanical engineer (E070) to industrial engineer (E060) would be $\mathrm{EE} 0706$.

HIREX Hiring variable representing hiring employees in an occupation. The variable representung hiring industrial engineers (EO60) would be HIREE 60 .

LAYOFX\# Layoff variable representing laying off of employees in an occupation. The variable representing laying off Mechanical Engineers (E070) would be HIREE 70.

AMINCOST The value of the objective function. 
CHIRE The sum of all hiring costs.

CLAYOF The sum of all layoff costs.

\section{Budget Model Variables}

$\mathrm{X}$ \#\#mber displaced. The variable representing the number of mechanical engineers (E070) to RIF would be E 70.

YMAXM The value of the budget reduction constraint.

Y3MAX The value of the objective function.

\section{Type}

There are five variable types:

C Column - These represent the occupational variables associated with training, hiring, layoff, and displacement.

$\mathrm{N} \quad$ Not-equal-type row - Rows with this type represent the value of a constraint with a not equal relationship, such as the objective function.

L Less-than-type row - Rows with this type represent the value of a constraint with a less-than relationship. The value of the variable is the difference between the value of the righthand side of the constraint and the lefthand side of the constraint. This represents the sum of the slack variables.

G Greater-than-type row - Rows with this type represent the value of a constraint with a greater-than relationship. The value of the variable is the difference between the value of the righthand and the lefthand sides of the constraint. This represents the sum of the surplus variables.

E Equal-type row - Rows with this type represent the value of a constraint with an equality relationship. The activity of these variables should always be 0 , because the difference between the two sides of the equation must be 0.0 by definition.

\section{Basis Status}

The status of the variable relative to the basis. A variable with a value greater than 0 is in the basis. The possible basis statuses are

$L$ Non-basic at lower bound. Variable is not part of the solution.

** Basic and infeasible. Variable is part of the solution, but the solution is infeasible.

BS Basic and feasible. The variable is part of the solution and the solution is feasible.

UL Non-basic at upper bound. Variable is not part of the solution.

EQ Equality row. 


\section{Activity}

The value of the variable. For example, if the variable EE 0706 has a value of 3 , this means that part of the solution is to train three mechanical engineers (E070) to be industrial engineers (E060); if the variable HIREE 06 is three, part of the solution is to hire three industrial engineers (E060). Variables with a value of 0 are not part of the solution.

For the variables HIRE 01 and LAYOF 01, the activity represents the total hire and layoff costs.

\section{Cost}

The cost coefficient of the variable. The values for all of the type $C$ variables are specified in the input data files created by the user, WT_LP.WK1 and WB_LP.WK1.

For the objective function variable, this represents the optimal solution.

\section{Lower Activity}

The Lower Activity is the activity level that would result from a cost coefficient increase from the Cost to the Upper Cost. This means that, as the cost coefficient increases, it costs more to keep the variable in the solution. At the Upper Cost, a different variable becomes cheaper and will enter the solution, replacing all or part of the first variable's Activity. The Lower Activity represents the new level of the variable in the solution after the Upper Cost threshold is crossed. If this value is infinity, any increase in the variable's Unit Cost will not affect the basis, only the value of the objective function.

\section{Unit Cost Down}

Unit Cost Down is the change in the objective function per unit change in the Activity down to the Lower Activity. It also represents the amount the Cost must be changed to reach the Upper Cost threshold (Cost + Unit Cost Down = Upper Cost).

\section{Upper Cost}

The Upper Cost is the threshold where the current variable in the basis will become more expensive than another variable not in the basis. At the Upper Cost, a different variable, the Limiting Process Down, becomes cheaper and will enter the solution, replacing all or part of the first variable's Activity level.

\section{Limiting Process Down}

This is the variable or process that will replace a variable in the basis when the basis variable's cost increases beyond the Upper Cost. If the Cost equals the Upper Cost, the two variables could be switched without affecting the optimal value of the objective function. In this case, there are multiple optimal solutions.

In many cases, it is possible that two or more variables qualify as Limiting Process Down. Because the output report only lists one, the alternatives are not explicitly reported. One way to identify alternative Limiting Process Down variables is to look for variables that accomplish the same function and have the same cost coefficient. For example, if the cost to train a mechanical engineer to be an industrial engineer is the same as the cost to train a nuclear engineer to be an 
industrial engineer, then the two could be interchanged without affecting the optimal solution (depending on other constraints).

\section{Limiting Process Down Status}

\section{Same as Basis Status.}

\section{Upper Activity}

The Upper Activity is the activity level that would result from a cost coefficient decrease from the Cost to the Lower Cost. This means that, as the cost coefficient decreases, it costs less to keep the variable in the solution. At the Lower Cost, the current variable becomes cheaper than another variable in the basis, and the current variable will force the other variable out of the basis, replacing all or part of the second variable's Activity. The Upper Activity represents the new level of the variable in the solution after the Lower Cost threshold is crossed. If this value is minus infinity, any decrease in the variable's Unit Cost will not affect the basis, only the value of the objective function.

\section{Unit Cost Up}

Unit Cost Up is the change in the objective function per unit change in the Activity up to the Upper Activity. It also represents the amount the Cost must be changed to reach the Lower Cost threshold (Cost + Unit Cost Up = Lower Cost).

\section{Lower Cost}

The Lower Cost is the threshold where the current variable in the basis will become less expensive than another variable not in the basis. At the Lower Cost, a different variable, the Limiting Process Up, becomes more expensive and will leave the solution, augmenting the first variable's Activity level.

\section{Limiting Process Up}

This is the variable or process that will be replaced in the basis when the current variable's Cost decreases beyond the Lower Cost. If the Cost equals the Lower Cost, the two variables could be switched without affecting the optimal value of the objective function. In this case, there are multiple optimal solutions.

In many cases, it is possible that two or more variables qualify as Limiting Process Up. Because the output report lists only one, the altematives are not explicitly reported. One way to identify alternative Limiting Process Up variables is to look for variables that accomplish the same function and have the same cost coefficient. For example, if the cost to train a mechanical engineer to be an industrial engineer is the same as the cost to train a nuclear engineer to be an industrial engineer, then the two could be interchanged without affecting the optimal solution (depending on other constraints).

\section{Limiting Process Up Status}

Same as Basis Status. 


\section{Lower Bound}

The minimal value a variable may be assigned. This is explicitly defined by the user in the Set Budget parameters screen.

\section{Upper Bound}

The maximum value a variable may be assigned. This is explicitly defined by the user in the Set Budget parameters screen.

\section{Reduced Cost}

The change in the value of the objective function per unit increase in the righthand side value of the constraint.

\section{Right-hand Side}

The value of the right-hand side of a constraint.

\subsection{Interpreting Output}

This section uses an example model presented in Section 3.3.2 of the Workforce Transition Model for DOE-AL Non-Nuclear Reconfiguration (Stahlman and Lewis 1993) to illustrate the comprehensive output format. Table 4.3 shows the parameters used in the example. Table 4.4 shows the output from the XPRESS-MP output file corresponding to the data in Table 4.3 (unimportant columns are not shown).

Table 4.3. Example Output Interpretation Parameters ( $X 01=$ Engineering Technicians and X $02=$ Health Physics Technicians)

\begin{tabular}{|c|c|c|c|c|c|}
\hline COCS & Current & Projected & Hire Cost & Lavoff Cost & $\mathrm{X01}$ \\
\hline 01 & $\begin{array}{r}10 \\
8\end{array}$ & $\begin{array}{r}7 \\
13\end{array}$ & $\begin{array}{l}10000 \\
10000\end{array}$ & $\begin{array}{l}14000 \\
14000\end{array}$ & $\begin{array}{r}0 \\
34000\end{array}$ \\
\hline
\end{tabular}

Table 4.4. Initial LP Output File

\begin{tabular}{|c|c|c|c|c|c|c|c|c|c|c|c|}
\hline Name & Basis & Activityo & & L activity & U_cost dwn & Upper cost & Lim dn pro & U activity & U_cost_up & Lower_cost & Lim_up_pro \\
\hline $\mathrm{Cl}$ & EQ & -3 & 0 & $-1.00 \mathrm{E}+30$ & 14000 & 0 & & 0 & -14000 & 0 & $\mid \mathrm{T} 01$ \\
\hline C2 & EO & 5 & 0 & 0 & -10000 & 0 & $\mathrm{H} 02$ & $1.00 \mathrm{E}+30$ & 10000 & 0 & \\
\hline Clo & $\overline{\mathrm{BS}}$ & 92000 & -92000 & 92000 & $1.00 \mathrm{E}+30$ & 0 & & $5.80 \mathrm{E}+34$ & 1 & 0 & $\times 0201$ \\
\hline $\mathrm{X} 02011$ & II & 0 & 34000 & -3 & -58000 & $1.00 \mathrm{~B}+30$ & T 01 & $1.00 \mathrm{E}+30$ & 58000 & -24000 & \\
\hline $\mathrm{X} 01021$ & iI & 0 & 34000 & $-1.00 \mathrm{E}+30$ & -10000 & $1.00 \mathrm{~B}+30$ & & 3 & 10000 & 24000 & TO1 \\
\hline $\mathrm{HOI}$ & $\mathrm{LL}$ & 0 & 10000 & -3 & -24000 & $1.00 \mathrm{E}+30$ & T 01 & $1.00 \mathrm{E}+30$ & 24000 & -14000 & \\
\hline $\mathrm{HO} 2$ & BS & 5 & 10000 & 2 & 10000 & 20000 & $\times 0102$ & $1.00 \mathrm{E}+30$ & 24000 & -14000 & $1 \mathrm{~T} 02$ \\
\hline T 01 & BS & 3 & 14000 & -2 & 10000 & 24000 & $\mathrm{X} 0102$ & $1.00 \mathrm{E}+30$ & 24000 & -10000 & $\mathrm{H} 01$ \\
\hline T 02 & LL & 0 & 14000 & -5 & -24000 & $1.00 \mathrm{E}+30$ & IHO2 & $1,00 \mathrm{E}+30$ & 24000 & -10000 & \\
\hline
\end{tabular}


In Table 4.4, C1 and C2 represent the two mass balance equations and have a Basis value of EQ indicating an equality equation. The only two variables in the solution, or basis, are $\mathrm{H} 02$ and $T$ 01. This represents the solution of laying off three Engineering Technicians and hiring five Health Physics Technicians. C10 is the objective function and has a value of 92000 . This is equal to the Activity level of each variable times their corresponding Cost. Thus:

$$
3 * 14000+5 * 10000=92000
$$

where all other activities or costs are 0 .

The Name, Activity, and Cost columns are the only columns required to identify the solution. The remaining columns provide sensitivity analysis information. The Lower_cost indicates how much the Cost value for a parameter must decrease before the solution changes. Lowering a variable's Cost to its Lower_cost will cause the variable's Activity level to increase by U_activity. This is because that variable becomes less expensive than another variable at that point. The variable it will replace in the basis is given by Lim_up_pro. Also, U_cost_up is the amount Cost must be decreased before the solution changes (Cost - U_cost_up $=$ Lower_cost).

For example, the variable X 0102 has a Cost of 34000 and a Lower_cost of 24000 . This means that if the Cost of X 0102 is lowered to 24000 or lower, it will become part of the solution. The difference $(34000-24000=10000)$ is the U_cost_up value. The U_activity value, in this case 3 , will be the new Activity value for the variable if the Cost is lowered to the Lower_Cost. Furthermore, Lim_up_pro indicates that the source of the 3 Activity units is T 01. Berause the current Activity level for $\mathrm{T} 01$ is 3 , the new solution should produce a $\mathrm{T} 01$ Activity of 0. Table 4.4 shows the soiution if X 0102's Cost is reduced to 24000.

Table 4.5. LP Output File (with X 0102 Cost $=24000$ )

\begin{tabular}{|c|c|c|c|c|c|c|c|c|c|c|c|}
\hline Name & Basis & Activity & Cost & L_activity & $U_{-} \cos d_{2} d u$ & Upper cost & $\operatorname{Lim}_{n}$ pro & U activity & U costup & Lower cost & Lim_up_pro \\
\hline $\mathrm{Cl}$ & $E Q$ & -3 & 0 & -5 & 14000 & 0 & $\mathrm{HO}_{2}$ & of & -14000 & 0 & $\mathrm{X} 0201$ \\
\hline C2 & $\mathrm{EQ}$ & 5 & 0 & 3 & -10000 & 0 & $\mathrm{HO2}$ & $1.00 \mathrm{E}+30$ & 10000 & 0 & \\
\hline C10 & BS & 92000 & .92000 & 92000 & $1.00 E+30$ & 0 & & $4.80 \mathrm{E}+34$ & 1 & 0 & X 0201 \\
\hline$\times 0201$ & $\mathrm{LL}$ & 0 & 24000 & -3 & -48000 & $1.00 \mathrm{E}+30$ & $\mathrm{X} 0102$ & $1.00 \mathrm{E}+30$ & 48000 & -24000 & \\
\hline $\mathrm{X} 0102$ & BS & 3 & 24000 & $-1.00 \mathrm{E}+30$ & 0 & 24000 & 01 & 5 & 24000 & 0 & $\mathrm{HOl}$ \\
\hline $\mathrm{HOl}$ & 11 & 0 & 10000 & -3 & .24000 & $1.00 \mathrm{E}+30$ & $\times 0102$ & 3 & 24000 & -14000 & $\mathrm{HO}_{02}$ \\
\hline $\mathrm{HO} 2$ & BS & 2 & 10000 & $-1.00 \mathrm{E}+30$ & 24000 & 34000 & 101 & 5 & 0 & 10000 & 01 \\
\hline T 01 & $\mathrm{LL}$ & 0 & 14000 & -2 & 0 & $1.00 \mathrm{E}+30$ & $\mathrm{HO2}$ & 3 & 0 & 14000 & 0102 \\
\hline$T 02$ & LL & 0 & 14000 & -2 & -24000 & $1.00 E+30$ & LHO2. & $1.00 \mathrm{E}+30$ & 24000 & -10000 & \\
\hline
\end{tabular}

When X 0102's Cost is lowered to 24000, X 0102 enters the basis with an Activity level of 3 as expected. Also, the Activity level of $\mathrm{T} 01$ is reduced to 0 as expected. The new solution is to train three Engineering Technicians to be Health Physics Technicians and to hire two Health Physics Technicians. Notice that the value of the optimal solution is still 92000 . This implies that both solutions are feasible at this point and are interchangeable. Lowering the Cost of either X 0102 or $\mathrm{H} 02$, however, will make one or the other preferable. The fact that there are multiple solutions at this point is indicated by $\mathrm{H} 02$ 's Cost being equal to its Lower_cost. Whenever a variable's Cost is equal to its Lower_cost (Upper_cost), any decrease (increase) in its Costs will cause the solution to change. This is an important clue for identifying multiple solutions. 
The remaining variables in Table 4.5 have Lower_costs that are negative. This means that the Costs would have to be negative for the solution to change and this cannot happen because of the non-negativity constraints. Thus, variables with negative Lower_costs cannot be forced into the solution by lowing their Costs.

Analysis of raising Costs is similar to lowering Costs. The Upper_cost indicates how much the Cost value for a parameter must increase before the solution changes. Increasing a variable's Cost to its Upper_cost will cause the variable's Activity level to decrease by L_activity. This is because that variable becomes more expensive than another variable at that point. The variable it will be substituted by is given by Lim_dn_pro. Also, U_cost_dn is the amount Cost must be increased before the solution changes (Cost + U_cost_dn =Upper_cost).

Continuing the example using Table 4.5, the variable $\mathrm{H} 02$ has a Cost of 10000 and an Upper_cost of 34000 . This means that if the Cost of $\mathrm{H} 02$ is raised to 34000 or higher, it will leave the solution. The difference $(34000-10000=24000)$ is the $U_{-}$cost_dn value. The L_activity value, in this case $-1.00 \mathrm{E}+30$, will be the new Activity value for the variable if the Cost is raised to the Upper_Cost. An Activity of this negative magnitude is not permitted because of the non-negativity constraints. Thus, the largest decrease possible is the current Activity level of 2. Furthermore, Lim_dn_pro indicates that the decrease in Activity will be absorbed by H 01 . Because the current Activity level for $\mathrm{H} 01$ is 0 , the new solution should produce a $\mathrm{H} 01$ Activity of 2. Table 4.6 shows the solution if H 02's Cost is raised to 35000.

Table 4.6. LP Output File (with $\mathrm{H} 02$ Cost $=35000$ )

\begin{tabular}{|c|c|c|c|c|c|c|c|c|c|c|c|}
\hline Name & Basis & Activity & Cost & I scivity & U cost dwn & Upper cout & U Lim dn pro & U agtivity & U cost up & Lower cost & Lim up pro \\
\hline C1 & EQ & -3 & 0 & 5 & -10000 & 0 & $\mathrm{HO} 01$ & $1.00 \mathrm{E}+30$ & 10000 & 0 & \\
\hline$C 2$ & EQ & 5 & 0 & 3 & -34000 & 0 & $\mathrm{HO} 01$ & $1.00 \mathrm{E}+30$ & 34000 & 0 & \\
\hline Clo & BS & 140000 & $-1 E+05$ & 140000 & $1.00 \mathrm{E}+30$ & 0 & & $5.80 E+34$ & 1) & 0 & $x 0201$ \\
\hline X02011 & 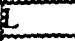 & 0 & 24000 & -3 & -48000 & $1.00 \mathrm{E}+30$ & $X 0102$ & $1.00 \mathrm{E}+30$ & 48000 & -24000 & \\
\hline $\mathrm{X} 0102 \mathrm{~B}$ & & 5 & 24000 & 3 & 1000 & 25000 & $\mathrm{HO}$ & $1.00 \mathrm{E}+30$ & 48000 & -24000 & $\mathrm{~T} 02$ \\
\hline $\mathrm{HOO}$ & BS & 2 & 10000 & -3 & 1000 & 11000 & $\mathrm{HO}$ & $1.00 \mathrm{E}+30$ & 24000 & -14000 & T01 \\
\hline $\mathrm{HOO2}$ & $L$ & 0 & 35000 & $-1.00 \mathrm{E}+30$ & -1000 & $1.00 \mathrm{E}+30$ & & 2 & 1000 & 34000 & $\mathrm{HOl}$ \\
\hline $\mathrm{TO}$ & $\mathrm{LL}$ & 0 & 14000 & -2 & -24000 & $1.00 \mathrm{E}+30$ & $\mathrm{HOI}$ & $1.00 \mathrm{E}+30$ & 24000 & -100000 & \\
\hline $\mathrm{TT} 02$ & $L$ & 0 & 14000 & -2 & -48000 & $1.00 \mathrm{E}+30$ & $\mathrm{HOl}$ & $1.00 \mathrm{E}+30$ & 48000 & 34000 & \\
\hline
\end{tabular}

When H 02's Cost is raised to 35000, $\mathrm{H} 02$ leaves the basis with an Activity level of 0 as expected. Also, the Activity level of $\mathrm{H} 01$ is increased to 2 as expected. The new solution is to train five Engineering Technicians to be Health Physics Technicians and to hire two Engineering Technicians. The value of the new optimal solution is 140000 . Such an increase in the hiring costs of Health Physics Technicians causes an increase of 48000 in the objective function.

Several other variables in Table 4.6 have Upper_costs that are $1.00 \mathrm{E}+30$, essentially infinite. This means that any increase in these variable's Costs will not affect the solution. That is because these variables are already the most expensive alternatives. Thus, variables with infinite Upper_costs cannot be forced out of the solution by raising their Costs; it will only make the solution more expensive. 


\subsection{Developing Scenarios and Performing Analyses}

This section describes the general formulation of the Workforce Transition Model linear program and how to define the input parameters to accurately reflect realistic transition strategies.

\subsection{Workforce Transition Model Formulation}

From the Workforce Transition Model for DOE-AL Non-Nuclear Reconfiguration report (Stahlman and Lewis 1993), the formulation of the Workforce Transition Model linear program is:

Minimize:

$$
\sum_{i=1}^{n} \sum_{\substack{j=1 \\ i \neq j}}^{n} X_{i j} x_{i j}+\sum_{i=1}^{n} H_{i} h_{i}+\sum_{i=1}^{n} T_{i} t_{i}
$$

such that:

$$
\begin{aligned}
& \sum_{\substack{j=1 \\
i \neq j}}^{n} x_{j i}-\sum_{\substack{j=1 \\
i \neq j}}^{n} x_{i j}+h_{i}-t_{i}=\Delta_{i} \quad i=1,2, \ldots, n \\
& x_{i j} \leq x_{i j, \max } \\
& x_{i j} \geq x_{i j, \min } \\
& h_{i} \geq h_{i, \min } \\
& h_{i} \leq h_{i, \max } \\
& t_{i} \geq t_{i, \min } \\
& t_{i} \leq t_{i, \max }
\end{aligned}
$$

where:

$$
\begin{aligned}
& \mathrm{i}, \mathrm{j}=\text { occupation indexes: } \mathrm{i}=1,2, \ldots, \mathrm{n} ; \mathrm{j}=1,2, \ldots, \mathrm{n} \\
& \Delta_{\mathrm{k}}=\mathrm{x}_{\mathrm{ib}}-\mathrm{x}_{\mathrm{ia}} \text {, or the change in the number of employees in occupation } \mathrm{i} \\
& \mathrm{x}_{\mathrm{ji}}=\text { number of employees training from occupation } \mathrm{j} \text { to occupation } \mathrm{i} \\
& \mathrm{x}_{\mathrm{ij}}=\text { number of employees training from occupation } \mathrm{i} \text { to occupation } \mathrm{j} \\
& \mathrm{h}_{\mathrm{i}}=\text { number of new hires in occupation } \mathrm{i} \\
& \mathrm{t}_{\mathrm{i}}=\text { number of employees in occupation } \mathrm{i} \text { terminated } \\
& \mathrm{X}_{\mathrm{ij}}=\text { cost of training an employee from occupation } \mathrm{i} \text { to occupation } \mathrm{j} \\
& \mathrm{H}_{\mathrm{i}}=\text { cost of hiring a person into occupation } \mathrm{i} \\
& \mathrm{T}_{\mathrm{i}}=\text { cost of laying off a person in occupation } \mathrm{i} \\
& \mathrm{x}_{\mathrm{ij}, \max }=\text { the maximum number of employees allowed to train from occupation } \mathrm{i} \\
& \mathrm{x}_{\mathrm{ij,min}}=\text { to occupation } \mathrm{j} \\
& \mathrm{h}_{\mathrm{i}, \min }=\text { the minimum number of employees allowed to train from occupation } \mathrm{i} \text { to } \\
& \mathrm{h}_{\mathrm{i}, \max }=\text { minimum number of new hires in occupation } \mathrm{i} \\
& \mathrm{t}_{\mathrm{i}, \min }=\text { maximum number of new hires in occupation } \mathrm{i} \\
& \mathrm{t}_{\mathrm{i}, \max }=\text { minimum number of layoffs in occupation } \mathrm{i}
\end{aligned}
$$


The parameters $x_{i, a}, x_{i, b}, x_{i j}, H_{i}, T_{i}, x_{i j, m i n}, x_{i j, m i n}, h_{i j, \min }, h_{i j, m i n}, t_{i j, m i n}$, and $t_{i j, \min }$ are the constants defined by the user either manually or through the WFTME. These are the values stored in WT_LP.WK1 (see Figure 2.1).

\subsection{Workforce Transition Model Scenario Development}

The WFTME is a tool that allows the user to define a transition strategy, run the strategy using the linear programming package, and then review the implications of the strategy. The lineur programming package will always find the optimal solution given the constraints defined by the user. The optimal solution, however, may not be realistic if the constraints are ill-defined. Developing an appropriate set of constraints is not always obvious initially, and an iterative approach is necessary to adequately define the problem. Thus it is important to understand what the optimal solution produced by the linear program means and to develop a strategy that will produce a well-defined set of constraints. This section provides general guidance for developing data files that accurately represent good transition stuategies.

\subsubsection{Initial Run - Unconstrained Baseline}

For the initial run, it is best to just define hinng costs, layoff costs, the current number of employees in each position, the projected number of employees in each position, and the training costs. All minimum values should be set to 0 , and all maximum values should be set to their maximum allowed value (9999 or 99999 ). The resulting value of the objective function provides a baseline to compare with other alternatives.

\subsubsection{Imposing Additional Constraints}

Running the baseline model will produce obvious extreme recommendations, because linear programs tend to extreme solutions if unconstrained. This means that, if using two or more resources to satisfy a constraint will yield the same optimal solution, the LP will choose all of one resource, and not a combination of equivalent resources.

For example, imagine that five managers are needed and that the costs for all engineering occupations to train to be managers are the same. Given no other constraints, there is no preference about which engineering occupations should be trained to be managers. The linear program would attempt to have all five trainees come from a single engineering occupation. This probably would be undesirable, because it would limit the turnover to a single occupation instead of distributing it among all possible occupations.

To compensate for the LP's extreme tendencies, minimum and maximum values can be imposed. Maximum values set an upper limit to the number that may train from one occupation to another, be hired, or be laid off. Maximum values do not require the related transition to be part of the solution. Minimum values require the specified transition to be part of the solution. If the minimum value is set to 3 , a minimum of three people must be involved in that transition.

To continue the example: if after the first run, the user is unsatisfied with all of the managers coming from a single engineering occupation, the maximurn for each engineering occupation training for management could be set at a number less than the five required. If 5 value is set to 2 from each engineering occupation, the next run of the LP would guarantee the five new managers be distributed among at least three engineering occupations. Alternatively, the minimum number 
required to train could be set greater than 0 for certain engineering occupations to control which occupations are forced into the solution.

Imposing minimum and maximum values also will impact other transitions because they limit or reserve resources. If there are only five industrial engineers, and a minimum number to lay off is defined as seven, an inconsistency arises. These inconsistencies may have two results. First, they may create an infeasible solution; the model becomes so constrained that no possible solution exists. Second, unrealistic transitions will arise. For the case of a minimum of seven industrial engineers being laid off when there are only five may result in two industrial engineers being hired and then immediately laid off to satisfy the constraint.

\subsubsection{Developing Alternative Scenarios}

Hiring costs, layoff costs, and the current workforce level are parameters that probably will not be changed. Projected workforce required, training costs, and minimum and maximum values are likely controllable parameters and should be part of the evaluation process.

Training costs may change depending on the installation's transition strategy. Training alternatives include courses available through local schools, in-house courses, or specially developed contracted courses. Alternative training options can be evaluated by running the model with different sets of training costs corresponding to the training alternatives.

Projected workforce is dependent on the future mission of the installation. A Contain and Control $(\mathrm{C} / \mathrm{C})$ alternative will require a more significant workforce than a Remove and Treat (R/T) alternative. Various cleanup strategies can be evaluated by changing the projected workforce characterization.

The minimum and maximum values are useful for tailoring the transition. At the extreme, the transition can be explicitly prescribed by setting the minimum values equal to the corresponding maximum values. This is a valid use of the model because, although the problem is explicitly defined, the linear program is able to calculate the cost of the transition or show that the transition is infeasible.

The output of the model will generally provide insight into which parameters need to be constrained. If the user is not comfortable with the results or if extreme solutions are identified, the minimum and maximum values of relevant parameters can be adjusted and the model rerun. The user should be careful not to constrain the model too quickly to avoid imposing the user's preconceived notions on the model. 


\subsection{Important Points}

This section describes some additional important points the user should be aware of that are not covered in preceding sections.

\subsection{Creating New Models}

Note that the models are configured to use only one set of file names, those in Tuble 1.2. To use multiple models, each model must be placed in a separate directory. Also, all of the WFTME supporting files must be resident in each model directory. To create a new model:

1. Create a new directory to place the new model.

2. Copy all of the .WK1 and .DBF files to the new directory.

3. Run the WFTME and use the Change Working Directory command to change to the new model directory. The WFTME will automatically read in the files and indicate if any files are missing.

Input files created in another application (for example, spreadsheet) may be used as the W? IN.WKI files as long as they follow the format specified in Figures 1.2 and 1.3 .

\subsection{Messages}

Messages are used extensively to assist the user. The two types of messages are Status Bar Messages and Window Messages.

Status Bar Messages: These messages appear in the lower lefthand comer in the status bar. They explain the function of the selected item and notify the user when the program is processing. Watch for these messages.

Window Messages: These messages appear as a gray window in the upper righthand corner. They are usually waming messages. Hit any key or click the mouse to continue. 


\subsection{References}

Holmes, C. W., R. E. Lewis, S. T. Hunt, and M. O. Finn. 1992. Environmental Restoration and Waste Management: U.S. Department of Energy Complex, Addendum. PNL-8171, Pacific Northwest Laboratory, Richland, Washington.

Stuhlman, E. J., and R. E. Lewis. 1993. Workforce Transition Model for DOE-AL Non-Nuclear Reconfiguration. PNL-8920, Pacific Northwest Laboratory, Richland, Washington.

XPRESS-MP User Guide. 1993. Dash Associates, Northants, United Kingdom. (Also available through Resource Optimization, Inc., Knoxville, Tennessee.) 


\section{Distribution}

No. of

Copies

OEESTCE

12 DOE/Office of Scientific and Technical Information
No. of

Coples

ONSTOE

DOERIahland Operntions Office

D.J. Sansotta

26 Pacific Northwest Laboratory

E.J. Stahlman (20)

Publishing Coordination

Technical Report Files (5)

Distr. 1 

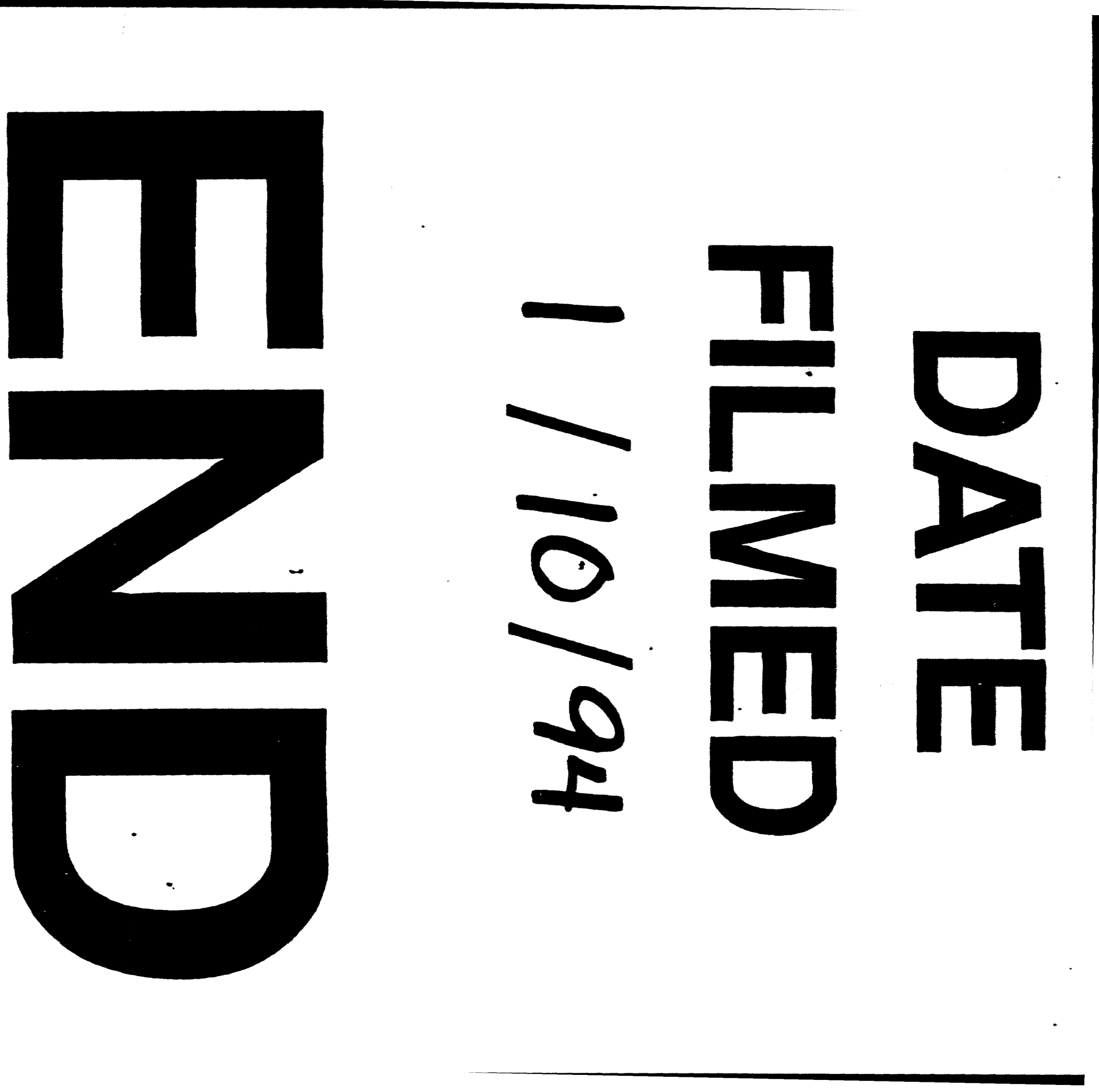
